\title{
ASSET POOLING, CREDIT RATIONING, AND GROWTH
}

\author{
Andreas Lehnert* \\ Board of Governors of the Federal Reserve System \\ Mail Stop 93 \\ Washington DC, 20551 \\ (202) 452-3325 \\ alehnert@frb.gov \\ December 8, 1998
}

\begin{abstract}
I study the effect of improved financial intermediation on the process of capital accumulation by augmenting a standard model with a general contract space. With the extra contracts, intermediaries endogenously begin using roscas, or rotating savings and credit associations. These contracts allow poor agents, previously credit rationed, access to credit. As a result, agents work harder and total economy-wide output increases; however, these gains come at the cost of increased inequality. I provide sufficient conditions for the allocations to be Pareto optimal, and for there to be a unique invariant distribution of wealth. I use numerical techniques to study more general models. Journal of Economic Literature classification numbers: O16, E44, G20, G33.

${ }^{*}$ The views are expressed are mine and do not necessarily reflect those of the Board of Governors or its staff. This paper is a substantially revised version of my dissertation. I thank Robert Townsend, Lars Hansen, Derek Neal, and Maitreesh Ghatak for several years of encouragement and support. I also thank Andrew Abel, Mitch Berlin, Ethan Ligon, Dean Maki, Steve Oliner, Wayne Passmore and Ned Prescott for helpful suggestions. I have also benefitted from the comments of seminar participants at the University of Chicago, UIC, Iowa State, Rice, Wharton, University of North Carolina, Tufts, University of Virginia, and the Federal Reserve Banks of Richmond, Philadelphia and Kansas City as well as the Board of Governors of the Federal Reserve System. Financial support from the University of Chicago, the Henry Morgenthau foundation and the Northwestern University/University of Chicago Joint Center on Poverty Research is gratefully acknowledged. Any remaining errors are mine.
\end{abstract}


Does financial intermediation directly cause growth, or is financial intermediation merely a necessary adjunct to growth? In this paper I identify a channel by which a nation's financial structure may directly affect its development experience. I augment a standard capital-accumulation model with a general contracting space. Armed with these extra contractual possibilities, financial intermediaries will endogenously arrange poorer agents into asset-pooling groups, which mimic one type of rosca (rotating savings and credit association) commonly observed in the developing world. Roscas help agents overcome credit rationing, increasing the demand for capital. The market-clearing interest rate increases, as does the average effort level. Output increases, but at the cost of increased inequality. Economies with the extra contracts grow faster to an invariant distribution of wealth with both a higher mean and greater inequality than economies without them.

The fact that financial intermediation, particularly asset-pooling contracts like roscas, contributes to inequality may be counter-intuitive. In my model there are two main reasons for this effect. First, asset-pooling groups cause the market-clearing interest rate to increase, thus increasing the premium to wealth. When the interest rate is higher, differences in wealth result in larger differences in consumption. Second, asset-pooling groups allow poor agents to leave their low-return, but safe, option for a highreturn, but risky, option. Because the market-clearing interest rate falls as wealth increases, these factors combine to produce Kuznets-style dynamics in the distribution of wealth, in which inequality is initially increasing and then decreasing. Note that the effect of wealth inequality depends 
crucially on the financial market structure. Without the asset-pooling contracts, inequality may reduce output. With them, I provide sufficient conditions for wealth inequality to have no effect on output at all.

The asset-pooling contracts that emerge endogenously may be interpreted as one-period roscas. Financial intermediaries will pool the wealth of many agents of the same wealth, assign the pool to a certain fraction of the contributors and then make them further loans (if needed). Because in this paper all agents of a given wealth will be identical and live for only one period, the pooled assets are divided with a lottery. Such contracts are known as lot roscas and are observed in the developing world, see for example the reviews of Besley, Coate and Loury $(1993,1994)$. Further, in a study of Mexican financial institutions, Mansell-Carstens (1993) finds that lot roscas are used by, among others, Volkswagon de Mexico's consumerfinance arm.

The extra contractual possibilities may also be interpreted as a joint stock company. All agents (of the same wealth) trade their wealth for one share in an enterprise jointly owned by them all. With the total equity from these shares, the enterprise either directly purchases capital inputs or approaches a bank for further debt financing of even more capital inputs. A certain proportion of the investors, chosen by lottery, are designated as managers. The enterprise allocates the accumulated capital to the managers for use in their projects. Agents, in my model, may only work on their own projects, and their labor effort is privately observed only by them. As a result, the correct level of labor effort is induced with an incentive-compatible 
"managerial compensation contract" that rewards those managers whose projects succeed and punishes those whose projects fail. The remaining shareholders, who were not selected as managers, become residual claimants and divide equally the output remaining after the managers are compensated and the bank repaid. From the point of view of an individual agent, an equity share in the enterprise represents a lottery ticket with a known probability of success. From the point of view of the enterprise as a whole, the probability that any one shareholder is designated a manager is the proportion whose projects may be funded.

Returning to the interpretation of input lotteries as roscas, Besley, Coate and Loury $(1993,1994)$ further find that roscas in general are Pareto-dominated by credit markets. In contrast, I provide sufficient conditions for allocations with asset-pooling contracts to be Pareto-optimal. This difference stems from the fact that in this paper, roscas emerge as an endogenous response to credit rationing, and are part of a larger credit system. The winners of the asset-pooling lottery may go on to get loans from financial intermediaries to augment their pooled assets. ${ }^{1}$

The model in this paper builds upon the work of Bannerjee and Newman (1993), Piketty (1997) and especially Aghion and Bolton (1997). These papers study the effect of, and the evolution of, the distribution of wealth in development. In this paper, I provide sufficient conditions for the distri-

\footnotetext{
${ }^{1}$ It is also worth noting that Besley, Coate and Loury consider multi-period roscas, in which agents must be prevented from defecting. In this paper, roscas last for one period only, as if agents could not be prevented from defecting.
} 
bution of wealth to converge to a unique invariant distribution, no matter what the initial distribution. Thus with the richer contract space there are no "poverty traps." Furthermore, with the extra contracts, I provide sufficient conditions for the distribution of wealth to have no effect on equilibrium prices or aggregate output. This difference stems from the fact that credit rationing provides the main mechanism, in those papers, by which distributions of wealth affect macroeconomic variables such as prices and output. In this paper, lottery based asset pooling contracts provide a mechanism to overcome credit rationing.

The analysis proceeds as follows: I define a contract space based on the work of Prescott and Townsend (1984a, b), in which contracts are seen as lotteries over possible outcomes. I then show how this abstract lottery space can be interpreted as a sequence of familiar contracts, and I solve analytically a model based on the work of Aghion and Bolton (1997). I then solve a set of richer models numerically, using a variant of the techniques of Phelan and Townsend (1991).

Section 1 below defines the notation, contract space and structure of the model. Section 2 specifies the equilibrium concept and some preliminary results about asset pooling. Section 3 presents analytic results from a model with risk neutrality and lumpy capital (fixed project size). I show that, in poor economies, asset-pooling lotteries increase output and the market-clearing interest rate; further, I provide sufficient conditions for optimality and a convergence result. Section 4 presents numerical results for a model with a richer technology and risk aversion. Section 5 concludes 
this paper.

\section{The Model}

In this section I describe the preferences, technology and endowments of agents in the model, the nature of the contracts which intermediaries offer to agents and how goods are stored from one period to the next. Here and for the rest of this paper, objects which are normally considered to be continuous (for example, consumption), are constrained to live in finite sets [following Prescott and Townsend (1984a, b)]. This provides simpler notation and analysis, and one can imagine allowing the number of points to grow arbitrarily large [see Phelan and Townsend (1991)].

\subsection{Preferences, Technology and Endowments}

Each agent lives for one period and produces exactly a single successor agent at the end of the period, towards which it is altruistic in a very special way: agents get utility directly from the amount bequested, not the utility value of bequests to the next generation. (This is often called "warm-glow" altruism.) Agents get a lump of consumption $\tau$ at the end of the period, where $\tau$ lies in the set $\mathbf{T}=\left\{\tau_{1}, \ldots, \tau_{n_{T}}\right\}$, and $\tau_{1}=0$, so that there is limited liability in the sense of Sappington (1983). Agents split this lump between own-consumption and bequests to their successor gen- 
eration. One can model this choice directly, but here I just assume that agents bequeath a constant fraction $s$ of their consumption lump $\tau$, and have indirect utility over $\tau$ given by $u(\tau)$, where $u^{\prime}>0, u^{\prime \prime} \leq 0$. While $\tau$ is public (that is, observed costlessly by all agents), the division between own-consumption and bequests is private (that is, observed only by the agent).

Agents also exert private labor effort, $z$ in $\mathbf{Z}=\left\{z_{1}, \ldots, z_{n_{Z}}\right\}$, where $z_{1}=0$. Private labor effort may be exerted on the agent's own technology only. Effort produces disutility of $\theta(z)$, where $\theta^{\prime}>0$ and $\theta^{\prime \prime} \geq 0$.

Agents then have preferences over consumption transfers $\tau$ in $\mathbf{T}$ and effort inputs $z$ in $\mathbf{Z}$ of:

$$
U_{\tau z}=u(\tau)-\theta(z) .
$$

All agents have access to a back-yard technology which maps inputs of private labor effort $z$ and public productive capital $k$ into a probability distribution over outputs $q$. Capital $k$ has to lie in the set $\mathbf{K}=\left\{k_{1}, \ldots, k_{n_{K}}\right\}$ (where $k_{1}=0$ ), and output $q$ has to lie in the set $\mathbf{Q}=\left\{q_{1}, \ldots, q_{n_{Q}}\right\}$. Both capital and output are public, and output may be costlessly confiscated (for example, by an intermediary). Inputs are timed so that capital is added first, before the agent decides on labor effort. Thus given inputs of effort $z$ and capital $k$, the technology, $P(q \mid z, k)$, specifies the probability of realizing a particular output $q$. In addition, for each possible input 
combination $z, k$ in $\mathbf{Z} \times \mathbf{K}$, the technology must satisfy:

$$
P(q \mid z, k) \geq 0, \text { and } \sum_{q} P(q \mid z, k)=1 .
$$

Capital is consumed entirely in the productive process. In the numerical section, the technology will have to satisfy the stricter condition $P(q \mid z, k)>$ 0 all $q, z, k$ in $\mathbf{Q} \times \mathbf{Z} \times \mathbf{K}$. This will prevent infinite likelihood ratios.

There is a continuum of agents of unit mass, a proportion $\psi^{a}$ of whom are endowed at the beginning of the period with one of $n_{A}$ levels of wealth $a$ in $\mathbf{A}=\left\{a_{1}, \ldots, a_{n_{A}}\right\}$, where for each $a, \psi^{a} \geq 0$ and $\sum_{a} \psi^{a}=1$. Wealth is in the form of capital, is public and may be costlessly transported among agents. Define $\psi$ to be the vector of population weights, $\left[\psi^{a_{1}}, \ldots, \psi^{a_{n}}\right]$.

\subsection{Contracts}

The contract space studied by Prescott and Townsend (1984a, b) uses lotteries to span non-convexities arising from moral-hazard constraints. For this reason, it is very useful in this paper. Contracts are specified as weights on the linear space of all of the possible combinations of consumption transfers, output, effort and capital, conditional on wealth. From the point of view of the economy as a whole, because there is a continuum of agents, the contract weights are fractions of agents who will receive a particular combination of consumption, output, effort and capital. From the point of view of a particular agent, they are the probability of receiving a particular combination. In section 3 below, I show how contracts in this abstract 
space may be interpreted in more familiar terms.

The space of valid contracts will be a linear space subject to some linear constraints. The constraints are, first, that the contracts form a valid set of probabilities; second, that they are Bayes compatible with the underlying technology; and third, that they are incentive compatible with respect to deviations in effort once capital has been announced. Let the linear space $L$ be the Euclidean space of dimension $n_{T} n_{Q} n_{Z} n_{K}$. A contract $x(\tau, q, z, k)$ must lie in the space $\mathbf{X}$, where: $\mathbf{X}=\{x \in L$, such that:

$$
\begin{aligned}
& x(\tau, q, z, k) \geq 0, \text { all } \tau, q, z, k ; \sum_{\tau q z k} x(\tau, q, z, k)=1 ; \\
& \sum_{\tau} x(\tau, \bar{q}, \bar{z}, \bar{k})=P(\bar{q} \mid \bar{z}, \bar{k}) \sum_{\tau q} x(\tau, q, \bar{z}, \bar{k}) \text { all }(\bar{q}, \bar{z}, \bar{k}) \text { in } \mathbf{Q} \times \mathbf{Z} \times \mathbf{K} ; \\
& \left.\sum_{\tau q} x(\tau, q, \bar{z}, \bar{k})\left[\frac{P(q \mid \hat{z}, \bar{k})}{P(q \mid \bar{z}, \bar{k})} U_{\tau \hat{z}}-U_{\tau \bar{z}}\right] \leq 0 \text { all } \bar{z}, \hat{z}, \bar{k} \text { in } \mathbf{Z} \times \mathbf{Z} \times \mathbf{K}\right\} .
\end{aligned}
$$

Because contracts can be viewed as joint lotteries over every possible combination of transfers $\mathbf{T}$, output $\mathbf{Q}$, effort $\mathbf{Z}$ and capital $\mathbf{K}$, the constraints (C1) to (C3) can be thought of as restrictions on those lotteries. In particular, equation (C1) requires that contracts form valid lotteries, that is, that they sum to unity and are non-negative. Equation $(\mathrm{C} 2)$ requires that the contracts respect the underlying probabilities given by the technology, $P(q \mid z, k)$. Finally, equation (C3) is the incentive-compatibility constraint; it requires that, for every assigned effort level $\bar{z}$, the agent not prefer some alternative effort, $\hat{z}$. For more on this constraint, see the appendix. 
Notice that the contract space $\mathbf{X}$ does not depend on the wealth $a$ of an agents. In the next section I introduce financial intermediaries who will offer the best possible contract in $\mathrm{X}$ to agents, subject to a zero-profit condition. This zero-profit condition will depend on agents' wealth.

Finally, it is convenient to assume that $\mathbf{A}=s \mathbf{T}$, so that if an agent is assigned the $j$-th element of $\mathbf{T}, \tau_{j}$, he bequeaths $s \tau_{j}$, which is (by assumption) exactly equal to $a_{j}$, the $j$-th element of A.

\section{Equilibrium and Pooling Contracts}

In this section I present the structure of financial intermediation, the equilibrium concept, and explanation of the three different types of lotteries used in the paper and some preliminary results on the use of pooling contracts.

\subsection{Competition in Financial Intermediation}

Zero-profit and costless financial intermediaries (called banks) will compete to attract depositors by offering the best possible contract that generates non-negative profits in expectation. The agent's assets are public and are placed completely on deposit with the intermediary. The capital input can be observed and controlled by the financial intermediary. Because agents live for only one period, contracts are single-period too. Both 
sides commit costlessly to the contract. Once contracts have been signed, the intermediary can confiscate output costlessly. Intermediaries take as given a risk-free rate of return on an interbank loan market. Because there is purely idiosyncratic uncertainty (which the banks smooth away by contracting with groups of agents), banks can borrow and lend freely at this rate on behalf of their depositors.

Because banks compete with one another to attract depositors, a bank will be unable to achieve zero profits by subsidizing the consumption of agents of one wealth type with the output of agents of some other wealth type. Banks attempting such a strategy would attract only the subsidized agent type. Financial intermediation, therefore, acts as if agents of each wealth type form a single bank, which makes zero profits. In the next section, I present sufficient conditions for this structure to yield the Pareto-optimal allocations. This will generally not be the case, because the social planner is not bound to respect a zero-profit condition on each wealth type. (See Holmström (1982) for the classic work on this topic. This effect can motivate a credit subsidy program for the poor.)

At the beginning of the period, one can imagine financial intermediaries writing complicated contracts combining insurance and production with agents. First, the intermediaries accept the agent's inherited stock of productive wealth on deposit, which they then place on the interbank loan market at rate $\rho$. Next, intermediaries determine how much capital the agent should be allocated. Because they can use input lotteries, this choice will be convex. Intermediaries borrow this amount on the interbank loan 
market. The contract also specifies how much the agent will be given to consume and bequeath conditional on inputs and outputs. If the agent is assigned some non-zero effort level, these consumption assignments will have to satisfy the incentive-compatibility constraint. Idiosyncratic uncertainty vanishes in the continuum, the intermediaries collect the output and distribute it among the agents in order to honor their commitments. Finally, agents consume and bequeath (by storage).

Let $W(a, \rho)$ be the expected utility of an agent of wealth $a$ when the interbank interest rate is $\rho$. Banks must compete to attract customers, so they offer contracts $y^{a}(\tau, q, z, k)$ to agents of wealth $a$ in $\mathbf{A}$ to maximize this expected utility:

$$
W(a, \rho) \equiv \max _{y^{a}(\tau, q, z, k) \in \mathbf{X}} \sum_{\tau q z k} y^{a}(\tau, q, z, k) U_{\tau z} .
$$

This maximization must proceed subject to the zero-profit constraint:

$$
\sum_{\tau q z k} y^{a}(\tau, q, z, k)(q+\rho a)-\sum_{\tau q z k} y^{a}(\tau, q, z, k)(\tau+\rho k) \geq 0 .
$$

As an accounting convention, the bank puts assets $a$ entirely on the interbank market, earning $\rho a$, and then borrows some amount $k$ on behalf of its agents, paying $\rho k$. Competition among banks will drive profits to zero. Notice that this zero-profit condition must hold for each wealth type $a$. The social planner would find it possible to violate these constraints for individual agent types, while respecting the resource constraint.

Market clearing requires that capital demanded for use as inputs must 
equal the aggregate wealth in the economy. Thus:

$$
\sum_{a} \psi^{a} \sum_{\tau q z k} y^{a}(\tau, q, z, k)(k-a)=0 .
$$

This, combined with the banks' zero-profit condition (2), implies that:

$$
\sum_{a} \psi^{a} \sum_{\tau q z k} y^{a}(\tau, q, z, k)(q-\tau)=0,
$$

which simply states that total consumption must equal total output.

Define an equilibrium as:

Definition Given an initial distribution of wealth $\psi_{0}$, an equilibrium is a sequence of interbank loan rates, contracts and wealth distributions:

$$
\left\{\rho_{t},\left\{y_{t}^{a}(\tau, q, z, k)\right\}_{a \in A}, \psi_{t}^{a}\right\}_{t=0}^{\infty},
$$

that satisfy the following conditions:

1. For all $t \geq 0, \rho_{t}$ and $\left\{y_{t}^{a}(\tau, q, z, k)\right\}_{a \in \mathbf{A}}$ satisfy:

(a) $0<\rho_{t}<\infty$.

(b) Given $\rho_{t}$, the set of period-t contracts $\left\{y_{t}^{a}(\tau, q, z, k)\right\}_{a \in \mathbf{A}}$ in $\mathbf{X}$ solve the bank's problem of maximizing the agent's expected utility, equation (1), subject to the non-negative profit condition, equation (2), for each agent type a in $A$.

(c) Bank profits are zero for each wealth type.

(d) At $\rho_{t}$ the interbank loan market clears, satisfying equation (3). 
2. For all $t \geq 0$, and for each wealth type, agents who receive a transfer of $\tau$ bequeath $s \tau$.

3. The mass of agents with wealth $a^{\prime}$ in period $t+1$ is given by:

$$
\psi_{t+1}^{a^{\prime}}=\sum_{a} \psi_{t}^{a} \sum_{q z k} y_{t}^{a}\left(\tau=a^{\prime} / s, q, z, k\right) .
$$

\subsection{The Nature of Lotteries}

There are three types of lotteries present in this model. The first, and least interesting type, are grid lotteries. These emerge as financial intermediaries attempt to span the discontinuities in the consumption vector, $\tau$. Grid lotteries appear only because variables that are normally taken as continuous are required to be discrete, and I attempt to minimize their effect by providing a dense grid over consumption in the numerical experiments. In section 3 below I assume that the grid is dense enough to be treated as an interval, so that grid lotteries disappear entirely. The second type are input lotteries. These are lotteries over assignments of capital and effort. At each outcome of these lotteries, there is a separate schedule of consumption assignments conditional on output, and, if a non-zero effort level is assigned, these must satisfy an ex post incentive compatibility constraint. The third and final type of lottery present is a standard equilibrium lottery formed as a convex combination of contracts and used to clear the market for capital.

In the discussion that follows I concentrate on the effect of the second of these three types: input lotteries. From the point of view of an individual 
agent, the contract specifies a probability, say $30 \%$, of realizing a particular capital level. From the point of view of a bank, the contract specifies what fraction of agents of a particular wealth will be allocated that capital level. These contracts allow the bank to concentrate the wealth of many poor agents into the hands of a subset, making them richer. This is tantamount to allowing banks to violate the zero-profit condition with respect to individual agents, while satisfying it for all agents of the same wealth. Thus these contracts allow intermediaries to pool the wealth of many agents and distribute the pool among a smaller group of agents of the same wealth.

An agent who loses the input lottery, or wins the input lottery but suffers the low output, is not necessarily consigned to zero consumption. There is scope in the contractual structure to allow ex post transfers back from the fortunate agents who won the input lottery and realized high output to those agents who either lost the input lottery or suffered the low output.

To study the effect of input lotteries, I briefly outline how to construct banks' optimal policies without them (more detail can be found in the appendix). Financial intermediaries are now restricted to contracts that assign inputs with $100 \%$ ex ante certainty. Let $W_{\mathrm{NL}}(a, \rho \mid z, k)$ be the utility of an agent with wealth $a$ when the interest rate is $\rho$ who is assigned inputs $(z, k)$ in $\mathbf{Z} \times \mathbf{K}$ with certainty. A financial intermediary offering this input combination chooses contracts $y_{\mathrm{NL}}^{a}(\tau, q \mid z, k)$ that satisfy:

$$
W(a, \rho \mid z, k) \equiv \max _{y_{\mathrm{NL}}^{a}(\tau, q \mid z, k)}-\theta(z)+\sum_{\tau q} y_{\mathrm{NL}}^{a}(\tau, q \mid z, k) u(\tau) .
$$

Contracts must satisfy versions of the constraints (C1) through (C3). These 
are detailed in the appendix. The maximization proceeds subject to the bank's zero-profit constraint:

$$
\rho(a-k)+\sum_{\tau q} y_{\mathrm{NL}}^{a}(\tau, q \mid z, k)(q-\tau) \geq 0 .
$$

Note that this constraint must hold separately for each $z, k$ combination.

Given values of $W_{\mathrm{NL}}(a, \rho \mid z, k)$ as determined above, for a borrower of particular wealth $a$ when the prevailing interest rate is $\rho$, the bank chooses an input combination $z, k$ such that:

$$
W_{\mathrm{NL}}(a, \rho)=\max _{(z, k) \in \mathbf{Z} \times \mathbf{K}} W_{\mathrm{NL}}(a, \rho \mid z, k) .
$$

The bank is forced to assign an input combination with certainty. It picks the one that produces the best utility for its borrower. Because $W_{\mathrm{NL}}(a, \rho)$ is formed from a constrained version of the maximization that produced $W(a, \rho)$, it must be the case that $W(a, \rho) \geq W_{\mathrm{NL}}(a, \rho)$. This construction leads directly to the observation that banks would always, if allowed, use input lotteries. Notice that the market-clearing price $\rho$ will be affected by the presence or absence of input lotteries. Controlling for these general equilibrium effects, it is not always necessarily the case that all agents will be made better off by the introduction of input lotteries. This point is considered in greater detail in the next section. 


\section{An Example with Lumpy Capital}

In this section I specify preferences, endowments and technology following Aghion and Bolton (1997), and solve analytically for the optimal contracts with and without input lotteries. Without input lotteries, the combination of moral hazard and limited liability [in the sense of Sappington (1983)] produces credit rationing. There is a threshold wealth required to get loans. This credit-market failure produces a non-convexity in the agent's expected utility. With the addition of input lotteries agents with wealth below the threshold can trade their wealth for a fair lottery over zero wealth and some high wealth above the threshold. I provide necessary conditions for this extra contract to increase the market-clearing interest rate, produce Pareto-optimal allocations, increase total economy-wide output and for the distribution of wealth to converge to a unique invariant distribution. The results in this section depend on several convenient assumptions, including risk-neutral agents and a special technology. Further, I do not here characterize the invariant distribution of wealth without lotteries. In the next section I use numerical techniques to compare the outcomes with and without input lotteries in a model which relaxes the assumptions on technology and preferences. In addition, I can compute the dynamics both with and without lotteries. 


\subsection{Economic Environment}

The economic environment is familiar: output $\mathbf{Q}$ can take on two values, $\{0,2\}$, where an output of zero means the project has "failed"; productive capital $\mathbf{K}$ is also limited to two values, $\{0,1\}$; while effort $\mathbf{Z}$ is assumed to be a dense grid on the interval $[0,1]$, so that contracts can be written essentially treating effort as continuous. Agents are risk neutral, so that in principle, transfers $\mathbf{T}$ could be limited to two values. However, to prevent grid lotteries from affecting the evolution of the distribution of wealth, transfers are also assumed to be densely gridded. The consumption transfer grid $\mathbf{T}$ is on the interval $[0,2 /(1-s \alpha)]$, where $\alpha$ is a preference parameter (see below) that will also turn out to be the highest market-clearing interest rate. Assets $\mathbf{A}$ are assumed to satisfy $\mathbf{A}=s \mathbf{T}$. The savings rate $s$ will also have to be constrained. See section 3.5 below. Finally, the choice of $k_{2}=1$ and $q_{2}=2$ is merely to conserve on notation. All of the following results go through with more general value for $k_{2}$ and $q_{2}$.

Agents have preferences that are linear in consumption transfers and quadratic in effort, so that:

$$
U_{\tau z}=\tau-\frac{z^{2}}{\alpha}, \text { where } 0<\alpha<1 .
$$

The technology exhibits strong complementarity between capital and labor effort:

$$
P(q=2 \mid z, k)= \begin{cases}z & \text { if } k \geq 1 \\ 0 & \text { if } k<1\end{cases}
$$




\subsection{Contracts}

An element $y$ of the contract space $\mathbf{X}$ can be interpreted as a probability mass function over all possible events in the economy that satisfies certain conditions. It is possible to rewrite the joint probability of a particular outcome $y(\tau, q, z, k)$ as a sequence of conditional probabilities. (The contract $y$ and all its component sub-lotteries in the discussion that follows are of course conditional on wealth $a$ and the interest rate $\rho$, but this notation is suppressed here for clarity.) Thus let $\xi$ be the probability of being assigned the high capital level. This can be formed from the underlying contract by integrating over all the other variables:

$$
\xi \equiv \sum_{\tau q z} y(\tau, q, z, k=1) .
$$

With probability $1-\xi$ the agent is not assigned capital. Given the extreme form of the technology, it makes no sense to assign him the high effort level because the low output is certain. Call $\tau_{0}$ the agent's assigned consumption in that case, so:

$$
\tau_{0} \equiv \sum_{\tau} \tau y(\tau, q=0, z=0, k=0) .
$$

Assume further that any desired $\tau_{0}$ is always an element of $\mathbf{T}$, so that, conditional on losing the input lottery [and thus the certain realization of low capital, low effort and low output $(k=0, z=0, q=0)$ ], the contract assigns a consumption transfer $\tau_{0}$ with certainty. This is the same as assuming that there are no grid lotteries required to realize an expected consumption transfer of $\tau_{0}$. 
If the agent is lucky (that is, is assigned the high capital input), then the agent will be assigned the high effort level with certainty. To see this, note that uncertainty over effort assignments would cost the agent utility (because $\theta^{\prime \prime}(z)>0$ ), would not increase expected output once capital had been assigned and would not help overcome the incentive compatibility constraint, because condition (C3) must hold after the resolution of any effort lottery. Therefore, conditional on assigning the high capital level, the bank will assign a single effort level with certainty, assuming that the the target effort is an element of $\mathbf{Z}$. For convenience, assume that this is always the case. (Even in the numerical work, it is possible to begin with one specification of grid elements, and then recursively adjust the grids by adding the expected value of any grid lotteries as an element of the grid.) Call the assigned effort $z_{\text {Icc. }}$ Thus:

$$
\begin{aligned}
& y\left(z=z_{\mathrm{ICC}} \mid k=1\right)=1, \text { and: } \\
& y\left(z \neq z_{\mathrm{ICC}} \mid k=1\right)=0 .
\end{aligned}
$$

Depending on the outcome of the project, the bank will transfer some amount of the consumption good to the agent. Define $\bar{\tau}$ to be the transfer conditional on the high output, $q=2$, and $\tau$ to be the transfer conditional on the low output, $q=0$, in the same way that $\tau_{0}$ was defined above. Once again, assume that $\mathbf{T}$ contains the right elements to avoid grid lotteries.

The choice of contract $y$ can thus be boiled down to a choice of the parameters $\left\{\xi, z_{\text {ICC }}, \tau_{0}, \bar{\tau}, \underline{\tau}\right\}$ for each wealth type $a$ in $\mathbf{A}$. To describe valid contracts, these parameters must satisfy conditions (C1) to (C3). The requirement 
that the contract be a valid lottery, (C1), can be satisfied if $0 \leq \xi \leq 1$, if $z_{\mathrm{ICC}}$ is in $\mathbf{Z}$. and if $\tau_{0}, \bar{\tau}, \underline{\tau}$ are all in $\mathbf{T}$. The requirement that the contract respect the underlying technology, $(\mathrm{C} 2)$, can be satisfied by requiring that:

$$
\begin{aligned}
\sum_{\tau} y\left(\tau, q=2, z=z_{j}, k=1\right) & =z_{j} \xi, \text { all } z_{j} \text { in } \mathbf{Z}, \text { and: } \\
\sum_{\tau z} y(\tau, q=2, z, k=0) & =0 .
\end{aligned}
$$

The final requirement is that effort assignments be incentive compatible. Using the incentive compatibility constraint (C3) above, it is easy to show that, if the agent has been assigned effort $z_{\mathrm{ICC}}$ and is contemplating a lower effort level, $\hat{z}<z_{\text {IcC, }}$, incentive compatibility requires that:

$$
z_{\text {ICC }} \leq \alpha(\bar{\tau}-\underline{\tau})-\hat{z} .
$$

Assume that the nearest point in $\mathbf{Z}$ less than $z_{\text {ICC }}$ is $z_{\text {ICC }}-h$. This is the largest possible deviation, $\hat{z}$. Thus the incentive compatibility constraint requires that:

$$
z_{\mathrm{ICC}} \leq \frac{\alpha}{2}(\bar{\tau}-\underline{\tau})+\frac{h}{2} .
$$

Assume that the grid over effort assignments $\mathbf{Z}$ is so dense that we can take $h$ to be zero. (Other interpretations are that the bank has to satisfy the "true" incentive compatibility constraint, formed when $h=0$, or is not exactly sure where the nearest grid point is.) Thus, given $\bar{\tau}$ and $\underline{\tau}$, the highest effort that may be assigned is:

$$
z_{\text {ICC }}(\bar{\tau}, \underline{\tau})=\frac{\alpha}{2}(\bar{\tau}-\underline{\tau}) .
$$


Notice that if the agent receives the project's payoff, so that $\bar{\tau}=2$ and $\underline{\tau}=0$, incentive-compatible effort is $z_{\mathrm{ICC}}=\alpha$, which is also the first-best effort level.

The agent's expected utility $w$ from a contract $\left\{\xi, z_{\text {ICC }}, \tau_{0}, \bar{\tau}, \underline{\tau}\right\}$ is:

$$
\xi\left[z_{\text {ICC }} \bar{\tau}+\left(1-z_{\text {ICC }}\right) \underline{\tau}-\frac{z_{\text {ICC }}^{2}}{\alpha}\right]+(1-\xi) \tau_{0} .
$$

Using the incentive compatibility condition (8) to substitute out $z_{\mathrm{ICC}}$ in terms of $\bar{\tau}, \tau$, this can be rewritten as:

$$
w\left(\xi, \bar{\tau}, \underline{\tau}, \tau_{0}\right)=\xi\left[\frac{\alpha}{4}(\bar{\tau}-\underline{\tau})^{2}+\underline{\tau}\right]+(1-\xi) \tau_{0} .
$$

Finally, the bank must satisfy a zero-profit condition. Assuming that the interbank interest rate is $\rho$, the bank's net revenues from a contract to an agent of wealth $a$ are:

$$
R\left(\xi, \bar{\tau}, \underline{\tau}, \tau_{0} \mid a, \rho\right)=2 \xi z_{\mathrm{ICC}}-\bar{\tau} \xi z_{\mathrm{ICC}}-\underline{\tau} \xi\left(1-z_{\mathrm{ICC}}\right)-(1-\xi) \tau_{0}-\rho \xi+\rho a,
$$

substituting $z_{\mathrm{ICC}}=\alpha(\bar{\tau}-\underline{\tau}) / 2$ gives:

$$
=\alpha \xi(\bar{\tau}-\underline{\tau})-\xi\left[\frac{\alpha}{2}(\bar{\tau}-\underline{\tau})^{2}+\underline{\tau}\right]-(1-\xi) \tau_{0}+\rho a-\rho \xi .
$$

Where the cost of capital, $\rho$, is multiplied by the probability of assigning capital (or the proportion of agents of wealth $a$ who are assigned capital), $\xi$, to calculate the cost of funds.

The general problem of the bank in this environment can be cast as choosing contracts (for each borrower type $a$ in $\mathbf{A}$ ) of $\left\{\xi(a), \bar{\tau}(a), \underline{\tau}(a), \tau_{0}(a)\right\}_{a \in \mathbf{A}}$ to maximize utility (9) subject to the condition that revenues, defined in equation (10), be zero for each borrower type. 


\subsection{Analysis Without Input Lotteries}

Prohibiting input lotteries is tantamount to forcing the bank to choose a level of $\xi$ of either 1 (capital assigned with certainty) or 0 (capital denied with certainty). If the bank chooses not to assign capital, then its maximization problem degenerates to:

$$
\max _{\tau_{0}} \tau_{0} \text {, subject to: } \rho a-\tau_{0} \geq 0 .
$$

The optimal value of the transfer, $\tau_{0}^{\star}(a)$, is clearly just $\rho a$, and assigned effort is zero. Thus write the agent's expected utility in this case as:

$$
W_{\mathrm{NL}}(a, \rho \mid \xi=0)=\rho a .
$$

If the bank assigns capital with certainty $(\xi=1)$ then it chooses contracts to maximize:

$$
\begin{aligned}
W_{\mathrm{NL}}(a, \rho \mid \xi=1)= & \max _{\bar{\tau}, \underline{\tau} \in \mathbf{T}} \frac{\alpha}{4}(\bar{\tau}-\underline{\tau})^{2}+\underline{\tau} \\
& \text { subject to: } \rho(a-1)+\alpha(\bar{\tau}-\underline{\tau})-\frac{\alpha}{2}(\bar{\tau}-\underline{\tau})^{2}-\underline{\tau}=0 .
\end{aligned}
$$

Because the smallest element of $\mathbf{T}$ is zero [there is limited liability in the sense of Sappington (1983)], for agents with wealth $a$ below the minimum capital scale $(a<1)$, the optimal transfer when the project fails, $\underline{\tau}^{\star}$, will be zero, and the optimal transfer when the project succeeds, $\bar{\tau}^{\star}$, will be less than the high output, 2. This in turn, through the action of the incentive compatibility constraint, equation (8), means that effort supplied under the contract, $z_{\mathrm{ICC}}$, will be below the first-best amount, $\alpha$. Thus the optimal 
transfer policies are:

$$
\begin{aligned}
& \underline{\tau}(a)= \begin{cases}0 & 0 \leq a \leq 1 \\
\rho(a-1) & a \geq 1,\end{cases} \\
& \bar{\tau}(a)= \begin{cases}1+\sqrt{1-\frac{2 \rho}{\alpha}(1-a)} & 0 \leq a \leq 1 \\
2+\rho(a-1) & a \geq 1 .\end{cases}
\end{aligned}
$$

From this, one can see that the supply of labor effort is:

$$
z_{\mathrm{ICC}}(a)= \begin{cases}\frac{\alpha}{2}\left[1+\sqrt{1-\frac{2 \rho}{\alpha}(1-a)}\right] & 0 \leq a \leq 1 \\ \alpha & a \geq 1 .\end{cases}
$$

These results also point towards the credit-rationing result of Aghion and Bolton (1997), namely, that a threshold wealth is required to obtain loans. For borrowers of wealth $a<1, \bar{\tau}(a)$ is real only if $a>a^{\star}(\rho)$, where the threshold wealth $a^{\star}(\rho)$ is:

$$
a^{\star}(\rho)=1-\frac{\alpha}{2 \rho} .
$$

Thus the expected utility of an agent who is assigned capital is:

$$
W_{\mathrm{NL}}\left(a, \rho \mid \xi=1, a \geq a^{\star}(\rho)\right)=\frac{\alpha}{4}\left[1+\sqrt{1-\frac{2 \rho}{\alpha}(1-a)}\right]^{2} .
$$

Agents with wealth below the threshold $a<a^{\star}$ cannot credibly commit to work hard enough to make a loan worthwhile at any interest rate.

This analysis implies a maximum and a minimum possible value for the market-clearing price, $\rho$. If $\rho \leq \alpha / 2$ then all agents will be assigned capital. Thus let $\rho_{\min }=\alpha / 2$. On the other hand, if $\rho \geq \alpha$ then even rich agents will be at best indifferent about operating the technology. Thus let $\rho_{\max }=\alpha$. 
Banks will assign capital to agents with wealth above the threshold required to get loans only if their expected utility is greater with capital. Thus the expected utility of an agent of wealth $a$, when the interest rate is $\rho$, in a world without asset-pooling lotteries, $W_{\mathrm{NL}}(a, \rho)$, is:

$$
W_{\mathrm{NL}}(a, \rho)= \begin{cases}W_{\mathrm{NL}}(a, \rho \mid \xi=0) & \text { if } a<a^{\star}(\rho) \\ \max \left\{W_{\mathrm{NL}}(a, \rho \mid \xi=0), W_{\mathrm{NL}}(a, \rho \mid \xi=1)\right\} & \text { if } a \geq a^{\star}(\rho) .\end{cases}
$$

Figure 1 plots the expected utility of an agent when input lotteries are prohibited, and also when they are allowed. (See the next section.) Notice the clear non-convexity in the expected utility schedule $W_{\mathrm{NL}}$ at $a^{\star}$ (here $a^{\star}=1 / 3$ ): with input lotteries, agents are able to convexify around this region.

\subsection{Analysis with Input Lotteries}

Input lotteries will allow banks to write contracts that allow poor agents that is, agents with wealth below the threshold required to get loans - access to capital. Banks will pool the assets of all agents of the same wealth and concentrate it in the hands of a selected subgroup. This subgroup will be chosen at random, because all agents are completely identical. This type of contract replicates the lot rosca studied by Besley, Coate and Loury $(1993,1994)$. The input lottery will smooth out the non-convexity in expected utility as a function of wealth, in a fashion identical to the "gambling for life" literature. ${ }^{2}$ Sadler (1998) studies a version of this problem,

\footnotetext{
${ }^{2}$ See Rosen (1997) for further references to this literature. It has long been understood that non-convexities, or "indivisibilities," in choice sets provide a motive for gambling.
} 


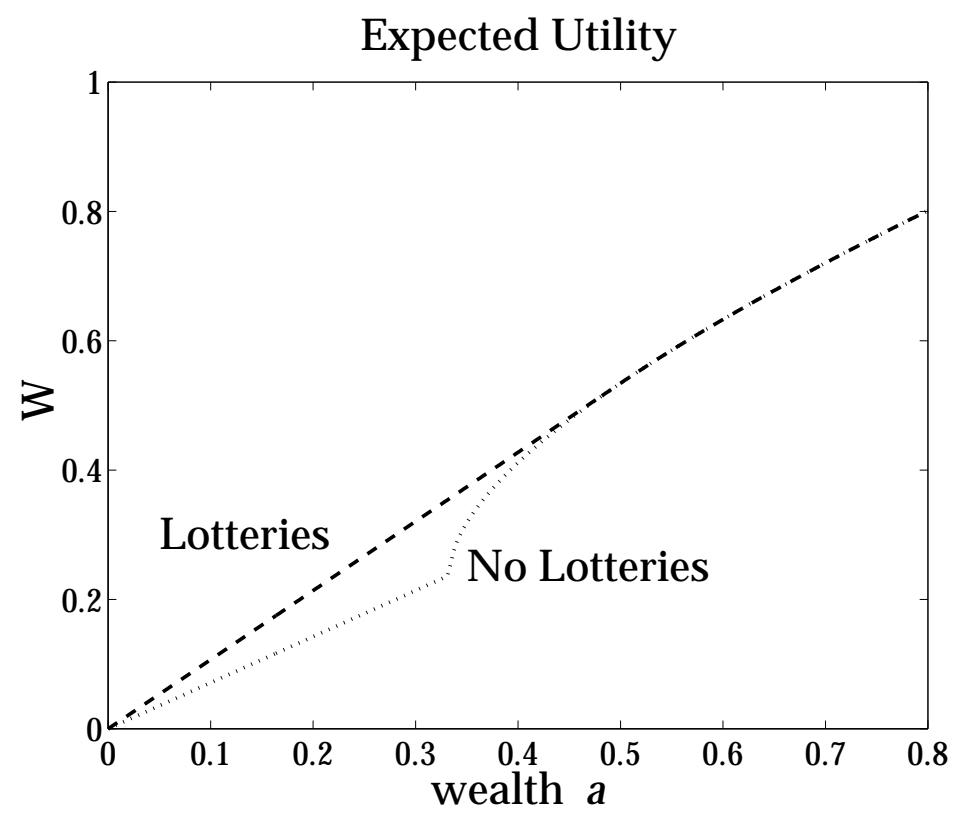

Figure 1: Expected utility with (dashed) and without (dotted) input lotteries when $\alpha=0.95$ and $\rho=$ 0.7125 . 
and shows that even risk-averse agents would be willing to take gambles when faced with credit-market non-convexities. Agents who are not credit rationed, and who would have been assigned credit anyway, will also avail themselves of these lotteries. In figure 1 these agents are located above the non-convexity but below the eventual target wealth.

The structure of the problem is the same as in the previous section, except that now banks may use a further control variable. As in the previous section, the non-negativity constraint on transfers will be binding for poor agents, so that agents with wealth below unity get a positive transfer only if they realize the high output. As before, $\underline{\tau}=0$. Without input lotteries, the transfer conditional on not being assigned the high capital level, $\tau_{0}$, was just $\rho a$. Now, with input lotteries, agents will prefer to concentrate all of their wealth into the state of the world in which they win the input lottery, so $\tau_{0}=0$. (This result depends on risk neutrality.) From equation (10), write the bank's revenue function as:

$$
R(\xi, \bar{\tau} \mid a, \rho)=\alpha \xi \bar{\tau}-\frac{1}{2} \xi \alpha \bar{\tau}^{2}+\rho a-\rho \xi .
$$

The bank's zero-profit condition is thus $R(\xi, \bar{\tau} \mid a, \rho)=0$. Divide both sides of this equation by the probability of being assigned capital, $\xi$, to form:

$$
\alpha \bar{\tau}-\frac{\alpha \bar{\tau}^{2}}{2}+\rho \frac{a}{\xi}-\rho=0 .
$$

The term $a / \xi$ can be thought of as the "target wealth" of the input lottery: all of the agent's wealth is concentrated into the state of the world in which In another context, firms that are in financial distress will undertake risky projects so that in some states, at least, they are not bankrupt. 
he wins the input lottery. The less likely this state is, the greater his wealth in it. Because $\xi$ is also the proportion of agents of wealth $a$ who win the input lottery, $a / \xi$ can also be thought of as the wealth transfer from the pooling group as a whole to those agents designated as managers.

By substituting in from equations (9) and (10) the bank's maximization problem is:

$$
\begin{aligned}
W(a, \rho)=\max _{\bar{\tau}, \xi \in[0,1]} \xi \frac{\alpha}{4} \bar{\tau}^{2}+(1-\xi) \cdot 0 \\
\quad \text { subject to: } \bar{\tau}^{2}-2 \bar{\tau}+\frac{2 \rho}{\alpha}\left(1-\frac{a}{\xi}\right)=0 .
\end{aligned}
$$

Another way to think about this problem is as a two-stage contract. In the first stage, banks concentrate a poor agent's wealth $a$ into an amount $a / \xi$ with probability $\xi$, and zero with probability $1-\xi$. In the second stage, the input lottery outcome has been realized, and there are two possibilities. Either the agent was lucky and won the input lottery, and now has wealth $a / \xi$, or the agent was unlucky and lost the input lottery and now has wealth zero. In either case, the bank then writes contracts with the agents as if there were no input lotteries. This tremendous simplification is entirely due to the assumption of risk-neutrality and limited liability. If agents were risk averse they would want insurance against the possibility of losing the input lottery, so $\tau_{0}$ could be non-zero, in which case this derivation does not go through. In the next section, I solve a range of numerical examples with risk-averse preferences and find similar differences between economies with input lotteries and economies without them. 
Thus the bank's problem (13) may also be written as:

$$
W(a, \rho)=\max _{\xi} \xi W_{\mathrm{NL}}(a / \xi, \rho \mid \xi=1)+(1-\xi) W_{\mathrm{NL}}(0, \rho \mid \xi=0) .
$$

The wealth variable $a$ and the choice variable $\xi$ can be replaced by the target wealth of the gamble, $a_{\mathrm{TARG}}=a / \xi$. From this it follows that the target wealth does not vary with own-wealth. Thus all agents who engage in an input lottery are seeking the same target wealth, poorer agents merely have a lower probability of achieving it.

Solving the bank's problem (13) above in terms of the target wealth, $a_{\text {TARG }}$ reveals that the optimal target is:

$$
a_{\text {TARG }}(\rho)=\frac{2 \rho}{\alpha}-1 .
$$

The associated lottery probability, $\xi(a, \rho)$, is $a / a_{\text {TARG }}(\rho)$. Notice that at the highest-possible interest rate, $\rho_{\max }$, the target wealth is unity, which is exactly the amount of capital required to operate the technology. At this interest rate, all agents with wealth $a \leq 1$ will be in a pooling group, and $\xi\left(a, \rho_{\max }\right)=a$, so they will use capital equal, in expected value, to their own wealth level. For all interest rates below $\rho_{\max }$, the target wealth will be below unity, so that even lucky agents in the asset-pooling group will still be net borrowers. Also, there will be some agents with wealth $a_{\text {TARG }}<a<1$ who will be net borrowers but will not be in a pooling group.

Banks will never assign rich agents (agents with wealth $a>1$ ) to pooling groups. As long as the interest rate satisfies $\rho \leq \rho_{\max }$, banks will set $\xi=1$ for rich agents. 
With this formulation, it is now possible to write down $W(a, \rho)$, the expected utility of an agent with wealth $a$ at interest rate $\rho$ when input lotteries are permitted. Thus:

$$
W(a, \rho)= \begin{cases}\left(a / a_{\mathrm{TARG}}\right) W_{\mathrm{NL}}\left(a_{\mathrm{TARG}}, \rho\right) & \text { if } 0 \leq a \leq a_{\mathrm{TARG}} \\ W_{\mathrm{NL}}(a, \rho) & \text { if } a \geq a_{\mathrm{TARG}} .\end{cases}
$$

Notice that $W(a, \rho)$ is linear in wealth below $a_{\mathrm{TARG}}$.

\subsection{Effect of Lotteries}

In the following results, I will require that the richest individual in the economy be unable to finance the project out of own funds. One can interpret this is as requiring that the economy be "poor" or that the project be large relative to household wealth. Let $a_{\max }$ be the wealth of the richest individual in the economy. In the propositions below, I require that $a_{\max } \leq 1$. I also provide sufficient conditions for the unique invariant distribution of wealth to satisfy $a_{\max } \leq 1$, so that all of these results will hold eventually.

\section{Proposition 1 (Interest Rate)}

For any distribution of wealth $\psi$ such that $a_{\max } \leq 1$, the market-clearing interest rate with input lotteries will be greater than the market-clearing interest rate without input lotteries.

Proof: See the appendix. 


\section{Proposition 2 (Aggregate Output)}

For any distribution of wealth $\boldsymbol{\psi}$ that satisfies $a_{\max } \leq 1$, the equilibrium aggregate output is higher with input lotteries than without them.

Proof: See the appendix.

\section{Proposition 3 (Pareto Optimality)}

For any distribution of wealth $\psi$ such that $a_{\max } \leq 1$, the equilibrium allocation with input lotteries produces utilities $\omega(a)$ that are Pareto optimal. The shadow value of capital of the social planner is the market-clearing equilibrium interest rate.

Proof: The proof is in the appendix. Although algebraically complex, it is conceptually straightforward: the equilibrium generates a set of expected utilities by wealth. When plugged into the social planner's problem as promised utilities, the social planner realizes a zero surplus.

Now consider the dynamics of this model. If the savings rate is above a critical level, the presence of lotteries merely accelerates growth to the same distribution studied by Aghion and Bolton (1997). In the complementary case, in which savings is low, it is a simple procedure to characterize the invariant distribution with lotteries. A subsistence technology (suppressed until now for expositional clarity) is required for nondegenerate dynamics. Without it, zero wealth becomes an absorbing state. Assume that any agent may completely abjure intermediation and place all capital and zero labor into a backyard technology in exchange for an $\epsilon$ probability of realizing the high output. This is merely the most conve- 
nient form of the subsistence technology. Nothing crucial depends on the assumption that own-capital is completely absorbed by the subsistence technology.

\section{Proposition 4 (Convergence)}

If the savings rate $s$ satisfies $s \leq 1 / 2$ and if there is a subsistence technology as defined above, then, for any initial distribution of wealth, the equilibrium price converges to $\rho_{\max }$ and the distribution of wealth converges to $\psi^{\star}$, in which a proportion $\psi_{0}^{\star}$ have wealth $a=0$ and the remaining proportion $\psi_{1}^{\star}$ have wealth $2 s$, where:

$$
\begin{aligned}
& \psi_{0}^{\star}=\frac{1-2 \alpha s}{1-2 \alpha s+\epsilon}, \text { and }: \\
& \psi_{1}^{\star}=\frac{\epsilon}{1-2 \alpha s+\epsilon} .
\end{aligned}
$$

Proof: See the appendix.

\section{An Example with Risk Aversion}

In this section I solve numerically a model with risk averse agents and multiple input choices. I use a version of the linear programming-based techniques of Phelan and Townsend (1991) to solve for the competitive equilibria with and without asset-pooling lotteries. (See also Prescott (1998) for recent developments in this literature.) I can compare the resulting time paths of wealth heterogeneity and market clearing interest rates along the transitions to the steady-state to determine the effect of adding asset- 
pooling lotteries.

I find that asset-pooling lotteries cause faster growth to a higher steadystate aggregate capital level and invariant distributions of wealth which feature greater inequality.

Economies with lotteries are more unequal for a variety of reasons. First, for the same distribution of wealth, the market-clearing interest rate is higher, so that small differences in wealth translate into larger differences in average consumption. Second, agents are in general assigned higher effort with lotteries, so that the incentive compatability constraint requires a greater variation in consumptions conditional on output. Third, the lotteries themselves promote inequality directly by rewarding lucky agents and punishing unlucky agents. If, without lotteries, a class of agents are "poor savers", then they will consume and bequeath equally. If, by adding lotteries, that same class of agents enter a pooling group, then the lucky ones will consume more than the unlucky ones.

Economists have generally known that the complementarity between labor effort and capital affects optimal contracts. ${ }^{3}$ To study this effect, I specify the technology to be CES and vary the complementarity parameter. Generally, I find that richer agents wish to supply less effort than poorer agents. If capital and labor effort are complements, this means that poor agents should be assigned capital, while if they are substitutes,

\footnotetext{
${ }^{3}$ See, as only one example in a large literature, Dupor (1998). In the context of moralhazard constrained contracting, Lehnert, Ligon, and Townsend (1998) consider the effect of complementarity in a model in which capital is not accumulated.
} 
poor agents should not be assigned capital. Thus the capital allocation curve, that is, how assigned capital varies with wealth, depends critically on the technological complementarity between capital and labor. One interpretation of so-called trickle-down dynamics is that the capital allocation curve is, in poor economies, steeply upward sloping, while in developed economies it is flatter. Those dynamics arise here if capital and labor are substitutes.

If capital and labor are complements, then there may be trickle-up dynamics, in which the capital allocation curve slopes down, and flattens as the economy develops. That is, if capital and labor are complements, then in poor economies, a rich agent does no work, is assigned no capital and consumes the rental value of his wealth. That same rich agent, in a relatively richer economy with a lower risk-free rate, might, in contrast, be assigned effort and capital, and consume both his (lower) rental income and the proceeds of his productive process.

\subsection{Parameter Values}

Agents are risk-averse, with a utility function given by:

$$
U_{\tau z}=2 \sqrt{\tau}-\frac{1}{4} z .
$$

Effort is limited to two values, so that $\mathbf{Z}=\{0,0.9\}$. Output can also take on only two values, $\mathbf{Q}=\{0,2\}$. Capital can take on one of five values linearly spaced between 0 and $1, \mathbf{K}=\{0,0.25,0.50,0.75,1\}$. The technology is 
chosen to mimic a standard CES production function in expected value, with the added constraint the probability of success or failure never be too high or too low. Thus:

$$
\begin{gathered}
g_{\alpha}(z, k)=\left(z^{\alpha}+k^{\alpha}\right)^{1 / \alpha}, \text { and: } \\
P_{\alpha}(q=2 \mid z, k)=\left\{\begin{aligned}
0.05 & \text { if } g_{\alpha}(z, k) \leq 0.05 \\
g_{\alpha}(z, k) & \text { if } 0.05 \leq g_{\alpha}(z, k) \leq 0.95 \\
0.95 & \text { if } g_{\alpha}(z, k) \geq 0.95 .
\end{aligned}\right.
\end{gathered}
$$

As part of the numerical experiment, I calculate equilibrium sequences and transition paths with and without lotteries for eight different values of $\alpha$ :

$$
\alpha=\{-100,-1,-0.5,-0.1,0.1,0.5,1,100\} .
$$

Negative values of $\alpha$ mean that capital and labor effort are complements (both are required to realize high output), while positive values mean that capital and labor effort are substitutes (either can be used to realize the high output). The extreme values of $\alpha,-100$ and 100 approximate a perfect complements (Leontieff) technology and a super-substitutes technology. The choice of $\alpha=100$ is unusual and deserves explanation. As $\alpha$ grows, the technology converges to the maximum operator, so that:

$$
\lim _{\alpha \rightarrow \infty} g_{\alpha}(z, k)=\max \{z, k\} .
$$

This is a quasiconvex function, and is seldom used. It is useful here, however, because it allows agents to realize the high output with either a high capital or a high effort level. Under the more standard formulation of perfect substitutes, $\alpha=1$, the technology is:

$$
g_{\alpha=1}(z, k)=z+k .
$$


Notice that, although their marginal contribution to output is independent of the other input level, both capital and labor are required to make sure of the high output.

The savings rate is fixed at $s=0.3$. In the numerical work, I found it more convenient to have intermediaries assign utility conditional on outcomes. Each assigned utility has an associated transfer and bequest policy. I provided a grid of 81 linearly-spaced utility points. This is equivalent to having 81 nonlinearly-spaced transfer and bequest points, with a denser concentration of points near the low end of transfers (where the utility function is more curved). Because of computational constraints, richer specifications of the technology, which feature more effort and capital points, must come at the cost of a sparser grid over transfers $\mathbf{T}$. This introduces undesirable grid lotteries into the computed solutions. The choice of technology here sacrifices some measure of technological verisimilitude in favor of a very dense grid over consumption transfers, $\mathbf{T}$.

\subsection{Results}

I begin with a detailed analysis of the case when $\alpha=-0.5$ (a typical case), and then discuss the results across all values of $\alpha{ }^{4}$

In figures 2 and 3 I plot the evolution of the distribution of wealth with

\footnotetext{
${ }^{4}$ The equivalent results from all the other values of $\alpha$ are suppressed to save space. They are available to the interested reader.
} 
and without lotteries for the case when $\alpha=-0.5$; while in figures 4 and 5 I plot the evolution of the total amount of wealth and the market clearing interest rate in both economies. Both economies begin with all agents endowed with zero capital. Because the minimum probability of the high output is $0.05,5 \%$ of these agents get the high output, and the resulting output is distributed equally to all agents, because there is no moral hazard. Once there is a little bit of capital in the economy, differences begin to emerge between the lottery and the no-lottery economies. These differences are initially small but cumulative. The no-lottery economy remains relatively poor with a concentrated wealth distribution, while the lottery economy is richer, with a less concentrated wealth distribution. Notice that the market-clearing interest rate, in figure 5, is initially greater in the economy with lotteries. Eventually the lottery economy becomes so much richer than the no-lottery economy that the market-clearing interest rate in the lottery economy falls well below the no-lottery economy.

The invariant distributions of wealth arrived at by both economies are displayed in figure 6. In figure 7 I plot the Gini coefficient (a common scalar measure of inequality) over time for both economies. Without lotteries, inequality rises steadily as the economy converges to the invariant distribution. With lotteries, there is an early surge in inequality, which then peaks and moderates slightly. This effect is much more dramatic for other values of $\alpha$. When $\alpha=-1$, for example, the Gini coefficient peaks near 0.23 before falling to its steady-state level of 0.125 . This accords well with the Kuznets hypothesis about inequality over the development cycle. For a careful microeconomic decomposition of inequality over time in Thai- 
land, see Jeong (1998).

In figure 8 I plot the evolution of aggregate capital from several different initial distributions. These different initial distributions feature different average wealth levels. Two of them begin with more capital than the steady state, so over time capital falls. The distributions converge to the same invariant distribution.

Repeating this analysis for all values of $\alpha$ yields a steady-state capital level and market-clearing interest rate for each. These are displayed in figures 10 and 11. Notice that the solutions are close at the extreme values of $\alpha$, but differ markedly in between. Notice also that economies with substitutes technologies (high values of $\alpha$ ) are richer no matter what the financial structure. This is because the production possibilities set is larger when either capital or labor may be used to achieve the high output. In all cases the economy with lotteries features a higher steady-state aggregate wealth level than the economy without them.

In general, invariant distributions in economies with lotteries feature greater inequality than in economies without lotteries. In figure 9 I plot the Gini coefficients from the invariant distribution of wealth at each value of the complementarity parameter $\alpha$. Notice than in seven of the eight cases, the Gini measure of inequality is higher with lotteries than without them. Only when $\alpha=0.1$ is the lottery economy more equal than the no-lottery economy, and even there they are close. Note also that in most cases the Gini coefficients lie between 0.05 and 0.20 , well below the estimates of 
modern developed economies, which lie between 0.4 and 0.6.

Distribution with Lotteries $(\alpha=-0.5)$

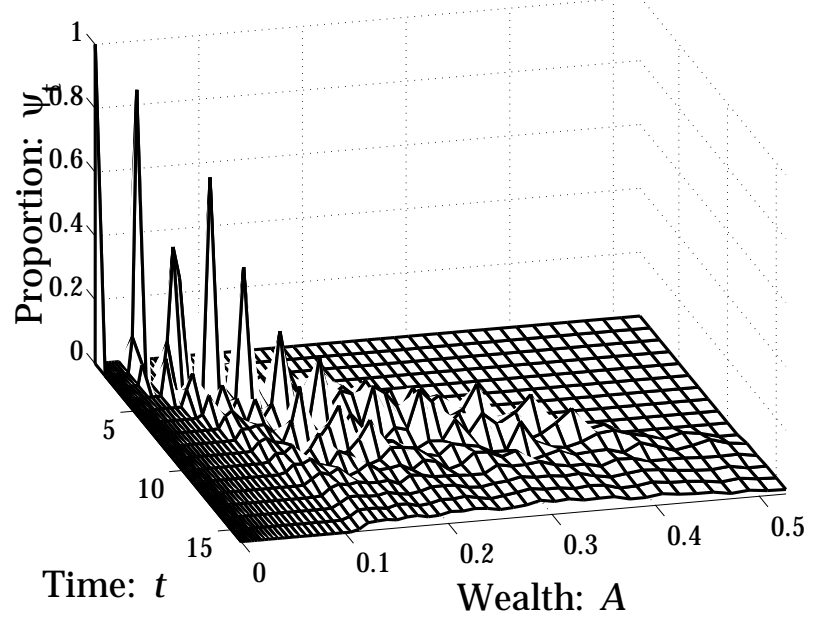

Figure 2: Evolution of the distribution of wealth with input lotteries.

Distribution, no Lotteries $(\alpha=-0.5)$

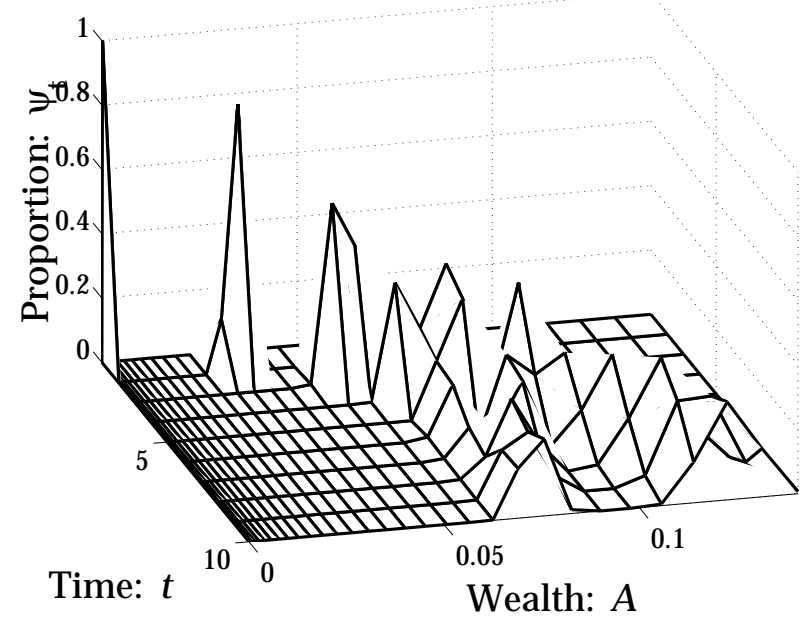

Figure 3: Evolution of the distribution of wealth without input lotteries. 


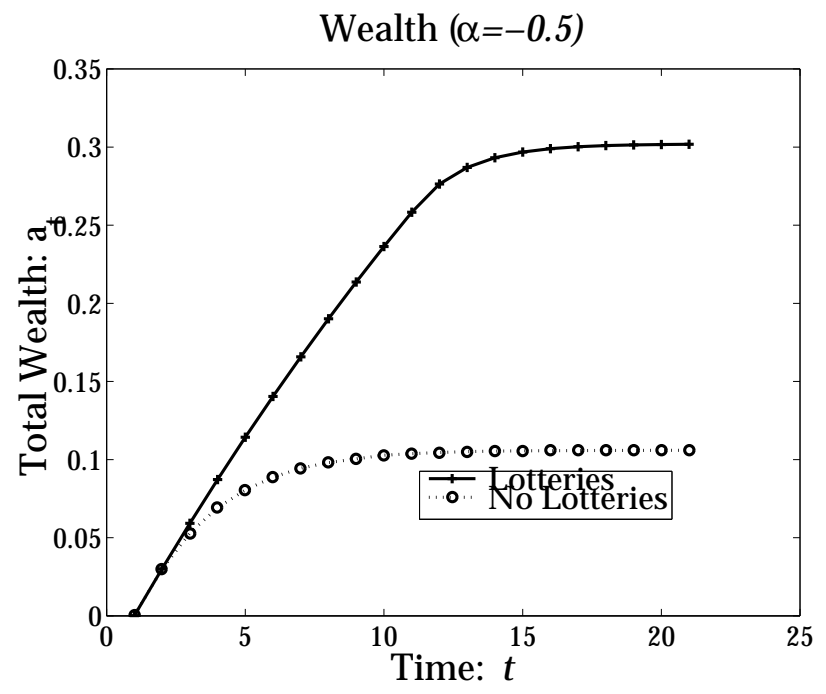

Figure 4: Evolution of aggregate wealth with and without input lotteries.

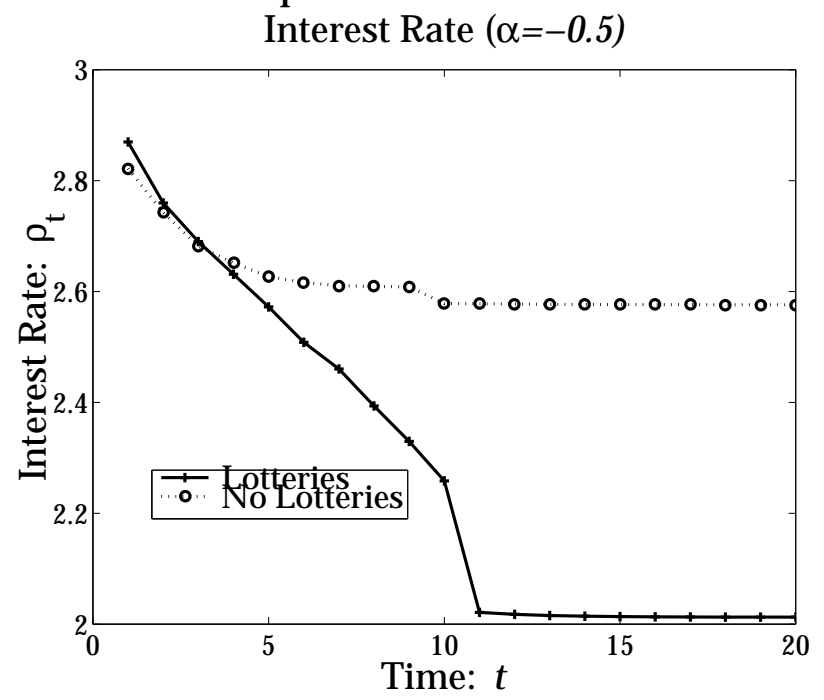

Figure 5: Evolution of market-clearing interest rate with and without input lotteries. 


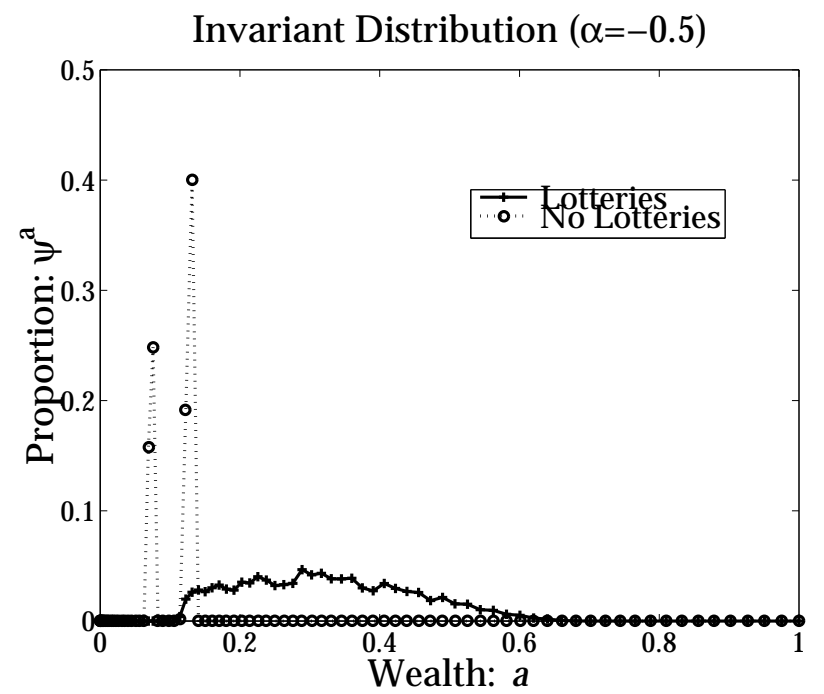

Figure 6: Invariant distributions of wealth with and without input lotteries.

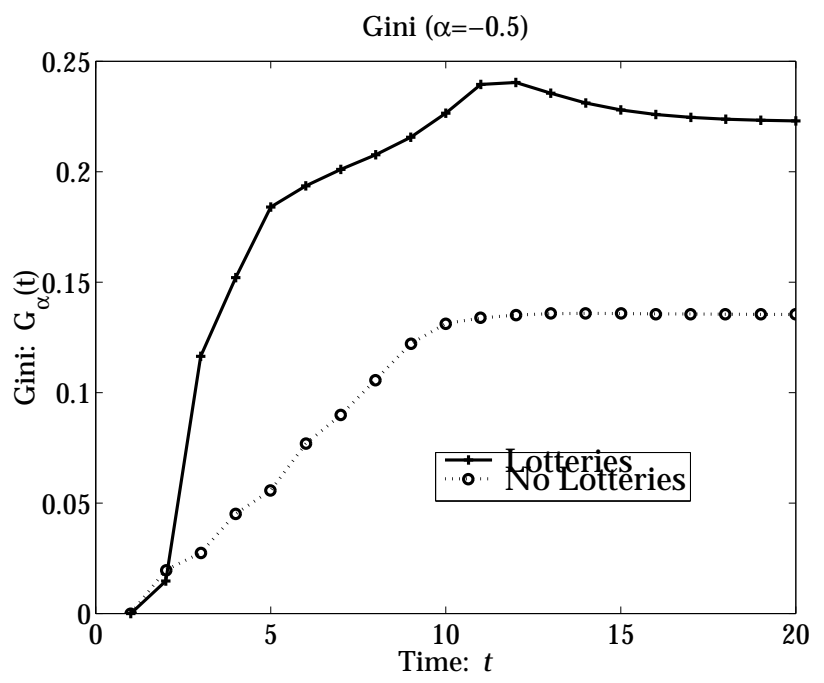

Figure 7: Evolution of the Gini coefficient over time, with and without input lotteries. 


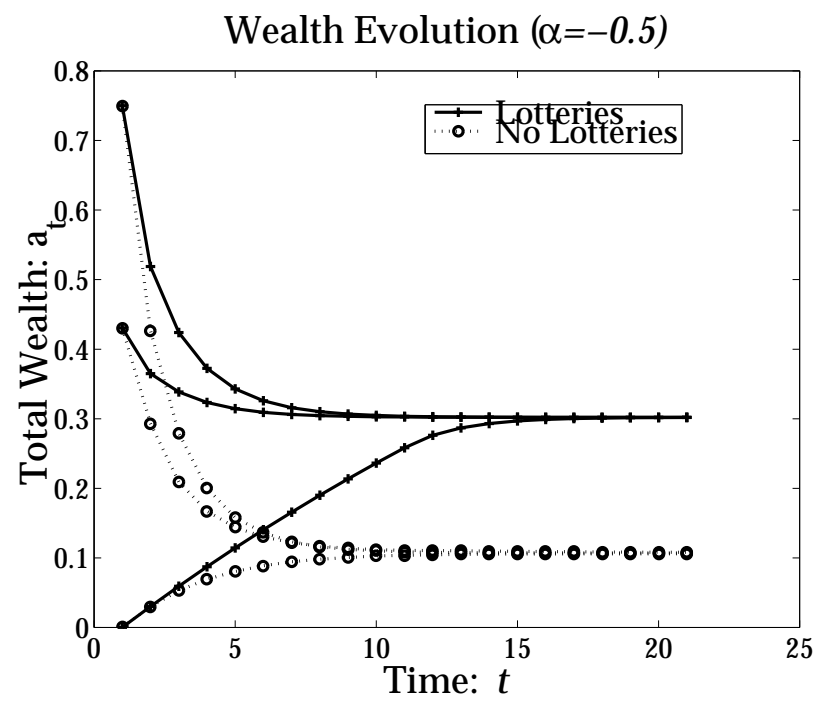

Figure 8: Aggregate capital levels over time from many different initial distributions.

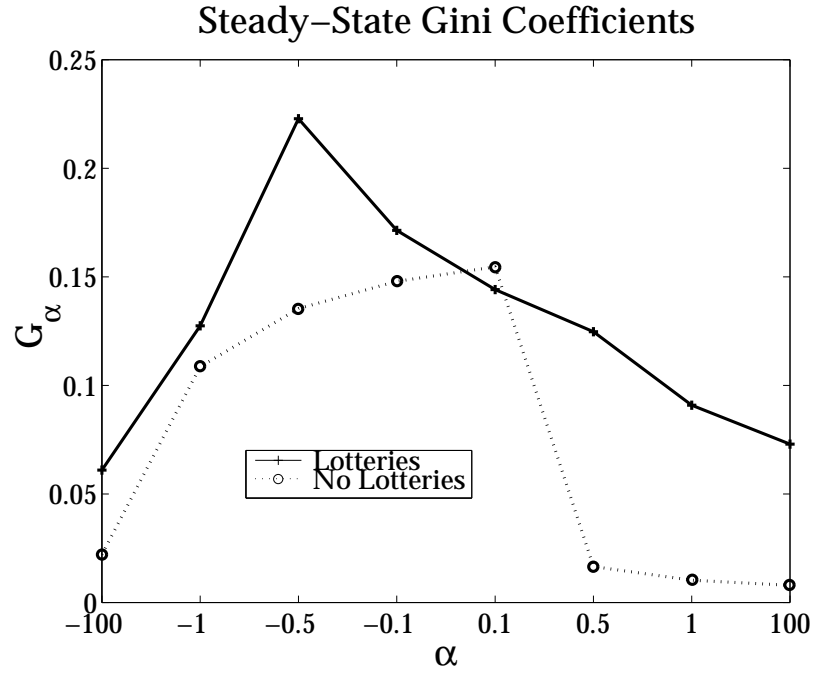

Figure 9: Gini coefficients of the invariant distributions of wealth with and without lotteries for different values of the complementarity parameter $\alpha$. 


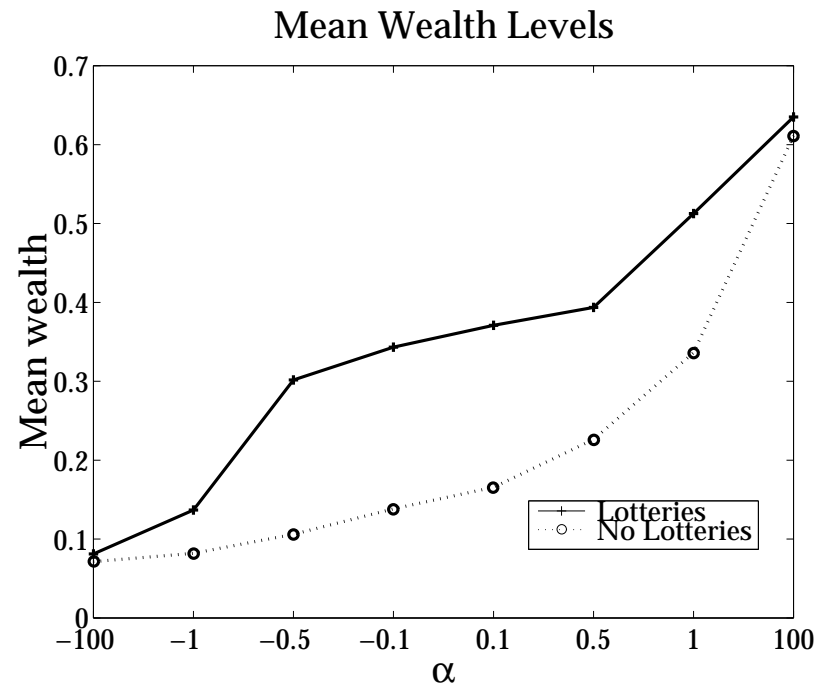

Figure 10: Terminal aggregate wealth levels with and without lotteries for different values of the complementarity parameter $\alpha$.

Terminal Interest Rates

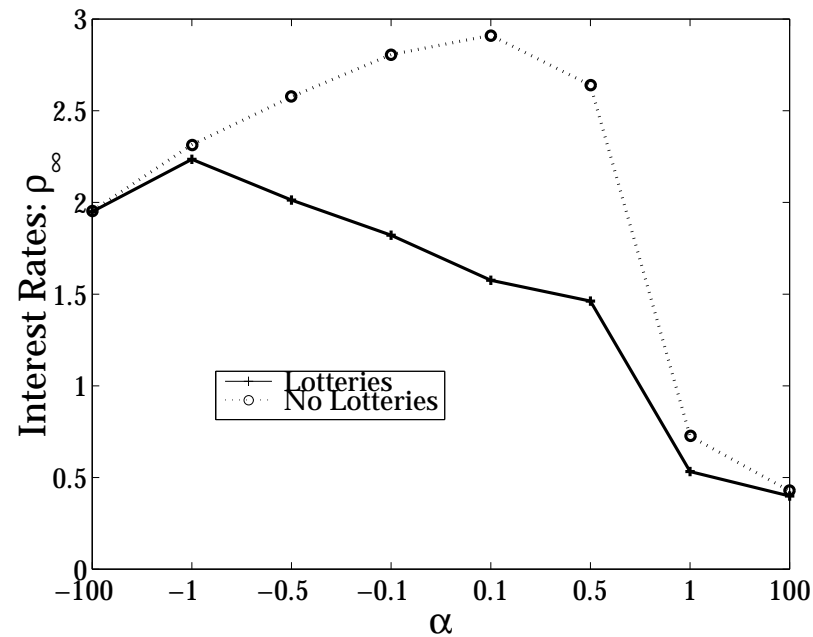

Figure 11: Terminal interest rates with and without lotteries for different values of the complementarity parameter $\alpha$. 


\section{Conclusion}

Group lending is usually taken to mean a joint-liability credit contract. This sort of lending is preferable because group members have an incentive to encourage others in their group to repay loans and may have means to pressure or monitor their peers not available to outside institutions. (See Ghatak and Guinnane (1998) for an excellent survey of this literature.) In this paper I have identified a subtle variant on this common and interesting contract. This paper concentrated on the ability of a group to pool its assets. The value of the summed assets is greater than the summed value of the assets because of non-convexities built into the technology and arising from endogenous credit rationing.

Most researchers agree that credit market imperfections play an important role in development. Recent models have focused on the productive costs of wealth inequality and the possibility that the poor might be trapped in poverty forever. This paper adopted the same general framework used in the literature (a capital market with a moral hazard problem) but allowed financial intermediaries to write very general contracts, based on lotteries, with borrowers. The asset-pooling contracts that then endogenously emerged closely resemble roscas, or rotating savings and credit associations.

This contractual innovation produced a host of interesting results. Lotteries interacted with credit markets to allow poor agents to escape the effects of credit rationing, and the invariant distributions of wealth featured 
a higher mean but also increased inequality with lotteries. Input lotteries could produce Pareto optimal outcomes, but not necessarily Pareto dominate allocations without lotteries.

How can we use these results in thinking about economic development? Because lotteries act as a pooling device, this paper can be thought of as discussing the consequences of pooling mechanisms in development. There is plenty of evidence that roscas play an important role in developing societies. Similar institutions, among them the familiar building societies, played an important role during the industrial revolution in developed countries [see Landes (1969)]. Alternatively, these results can be thought of as pointing towards the effect of better financial intermediation.

Given the structure of competition among financial intermediaries, this paper featured no barrier to contracts other than an endogenous moral hazard constraint. Pooling contracts could be victims of a host of other problems: they could be prohibited by government fiat (perhaps for domestic political reasons); some cost to financial contracting (not modeled in this paper) could further constrain contracts between borrowers and lenders; or there could be a commitment problem, with either agents or intermediaries allowed to renege on their obligations. When examining institutions as they exist in developing countries we have to keep this list of calamities in mind.

Presented with two otherwise identical nations, differing only because in one (for the reasons outlined above) asset-pooling groups do not exist, 
while in the other nation they do exist, we would expect the former nation to grow more slowly and settle down to a lower capital level than the latter nation. The nation without pooling groups, however, would feature less inequality than the nation with pooling groups. 


\section{Appendix}

\section{The Incentive Compatibility Constraint}

The incentive compatibility constraint in the contract space $\mathbf{X},(\mathrm{C} 3)$, can be derived following Prescott and Townsend (184a,b) and Phelan and Townsend (1991). One can think of the contract as specifying a conditional sub-lottery over consumption $\tau$ upon the realization of output $q$ with probabilities $x(\tau \mid q)$. Thus for expected utility given an assigned effort $\bar{z}$ and capital $\bar{k}$ to dominate the expected utility from a contemplated deviation in effort to $\hat{z}, x(\tau \mid q)$ must satisfy:

$$
\sum_{\tau q} x(\tau \mid q) P(q \mid \bar{z}, \bar{k}) U_{\tau \bar{z}} \geq \sum_{\tau q} x(\tau \mid q) P(q \mid \hat{z}, \bar{k}) U_{\tau \hat{z}}
$$

This may be rewritten as:

$$
\sum_{\tau q} x(\tau \mid q) P(q \mid \bar{z}, \bar{k}) U_{\tau \bar{z}} \geq \sum_{\tau q} x(\tau \mid q) P(q \mid \bar{z}, \bar{k}) \frac{P(q \mid \hat{z}, \bar{k})}{P(q \mid \bar{z}, \bar{k})} U_{\tau \hat{z}} .
$$

Multiplying by the marginal probability of a particular assignment $(\bar{z}, \bar{k})$ produces:

$$
\sum_{\tau q} x(\tau, q, \bar{z}, \bar{k}) U_{\tau \bar{z}} \geq \sum_{\tau q} x(\tau, q, \bar{z}, \bar{k}) \frac{P(q \mid \hat{z}, \bar{k})}{P(q \mid \bar{z}, \bar{k})} U_{\tau \hat{z}} .
$$

Which is, of course, exactly the constraint in equation (C3) above.

There are a few subtleties to the incentive compatibility constraint as used in this paper. The order of inputs, for example, makes a critical difference. The model assumes that capital is applied before effort, so that the agent knows $k$ before selecting $z$. If capital $k$ were selected after effort $z$, so that the agent could only know the distribution of possible values of capital when choosing effort, then the incentive compatibility constraint would be:

$$
\sum_{\tau q k} x(\tau, q, \bar{z}, k) U_{\tau \bar{z}} \geq \sum_{\tau q k} x(\tau, q, \bar{z}, k) \frac{P(q \mid \hat{z}, k)}{P(q \mid \bar{z}, k)} U_{\tau \hat{z}} .
$$

When choosing $z$, with $k$ not known, the agent must use the contracted probability distribution $x$ in determining the expected utility values of various plans. Notice that, because there are now only $n_{Z}^{2}$ constraints, as opposed to $n_{Z}^{2} n_{K}$ in 
(C3) above, $\mathbf{X}$ would be a larger set. The extra choices lead to solutions that are weakly better. On the other hand, it seems more natural to have effort supplied conditional on a particular capital input to the technology, and it is the usual specification in the literature.

Because the capital input is public, the suggested capital input level does not need to be induced. If, however, capital were private, so that, for example, agents were free to reinvest any capital transfers anonymously in banks before the resolution of production uncertainty, then suggested capital levels would have to be induced. The benefit to deviations in capital level would be purely pecuniary. Optimal contracts subject to this "input diversion" constraint are studied in much greater detail by Lehnert, Ligon, and Townsend (1998), who find that it can dramatically alter input use.

\section{Contracts Without Input Lotteries}

Let $W_{\mathrm{NL}}(a, \rho \mid z, k)$ be the expected utility of an agent with wealth $a$ when the market-clearing interest rate is $\rho$, who is assigned input combination $(z, k)$ with certainty. Let $W_{\mathrm{NL}}(a, \rho)$ be the expected utility of an agent when the bank has chosen the best input combination $(z, k)$. Thus he bank chooses contracts $y_{\mathrm{NL}}^{a}(\tau, q \mid z, k)$ to solve:

$$
W_{\mathrm{NL}}(a, \rho \mid z, k) \equiv \max _{y_{\mathrm{NL}}^{a}(\tau, q \mid z, k)}-\theta(z)+\sum_{\tau q} y_{\mathrm{NL}}^{a}(\tau, q \mid z, k) u(\tau)
$$

The maximization proceeds subject to the bank's zero-profit constraint:

$$
\rho(a-k)+\sum_{\tau q} y_{\mathrm{NL}}^{a}(\tau, q \mid z, k)(q-\tau) \geq 0 .
$$

Note that this constraint must hold separately for each $z, k$ combination.

The distribution over outputs is determined by the choice of non-stochastic inputs. Thus for each $\bar{q}$ in $\mathbf{Q}$, given a choice of inputs $\bar{z}, \bar{k}$, the Bayes compatibility constraint $(\mathrm{C} 2)$ becomes:

$$
\sum_{\tau} y_{\mathrm{NL}}^{a}(\tau, \bar{q} \mid \bar{z}, \bar{k})=P(\bar{q} \mid \bar{z}, \bar{k}), \text { all } \bar{q}, \bar{z}, \bar{k} \text { in } \mathbf{Q} \times \mathbf{Z} \times \mathbf{K} .
$$


Finally, the assigned effort must be incentive-compatible, so that if inputs $\bar{z}, \bar{k}$ are assigned, the contract $y_{\mathrm{NL}}^{a}(\tau, q \mid \bar{z}, \bar{k})$ must satisfy, for all possible deviations $\hat{z}$ in $\mathbf{Z}$ :

$$
\begin{aligned}
\sum_{\tau q} y_{\mathrm{NL}}^{a}(\tau, q \mid \bar{z}, \bar{k})\{u(\tau)-\theta(\bar{z})- & \\
& \left.\frac{P(q \mid \hat{z}, \bar{k})}{P(q \mid \bar{z}, \bar{k})}[u(\tau)-\theta(\hat{z})]\right\} \geq 0, \text { all } \bar{q}, \bar{z}, \bar{k} \text { in } \mathbf{Q} \times \mathbf{Z} \times \mathbf{K} .
\end{aligned}
$$

Notice that there may be no contract $y_{\mathrm{NL}}^{a}(\tau, q \mid z, k)$ for a particular combination $z, k$ that satisfies conditions (6), (15) and (16). If this is the case, let $W_{\mathrm{NL}}(a, \rho \mid z, k) \equiv$ $-\infty$. Clearly, there is at least one contract that does satisfy conditions (6), (15) and (16), namely, one that assigns the lowest effort and capital level, $z=0, k=0$ and has transfers that equal the output realizations, $\tau=q$.

\section{Proof of Proposition 1}

The equilibrium with lotteries is easy to calculate. At $\rho$ the aggregate demand for capital with lotteries is:

$$
K^{d}(\rho)=\sum_{a} \psi^{a} \xi(a, \rho)
$$

That is, if banks assign a proportion $\xi(a, \rho)$ of each wealth type capital, then the aggregate demand for capital is the weighted sum of the proportions. From equation (14) above, it is clear that $a_{\text {TARG }}\left(\rho_{\max }\right)=1$, so $\xi\left(a, \rho_{\max }\right)=a$. Thus, with lotteries:

$$
K^{d}(\rho)=\sum_{a} \psi^{a} a
$$

But this is just the aggregate quantity of capital in the economy. Further, if $\rho<$ $\rho_{\max }$, then $\xi(a, \rho)>a$, so the aggregate demand exceeds aggregate supply.

Without lotteries, in contrast, when $\rho=\rho_{\max }$ no agents with wealth $a<1$ will operate the technology. If there is any capital in the economy then the capital market has not cleared. Thus the equilibrium interest rate without lotteries must be strictly less than $\rho_{\max }$ 


\section{Proof of Proposition 2}

The strategy here is to show that output with lotteries attains the first-best level, and that output without lotteries must fall short of this level. With lotteries, from proposition 1 , the equilibrium interest rate must be $\rho_{\max }$. Hence from equation (14) it is clear that the target wealth is $a_{\text {TARG }}\left(\rho_{\max }\right)=1$ and the probability of winning the input lottery, $\xi\left(a, \rho_{\max }\right)$ is just $a$. From equation (12), it is clear that the effort assigned those agents who win the lottery will be $z_{\mathrm{ICC}}=\alpha$. Thus each agent has a probability $a \alpha$ of realizing the high output (in this case, 2). Hence aggregate economy-wide output with lotteries, $\bar{Q}$, is

$$
\bar{Q}=2 \alpha \sum_{a} \psi^{a} a
$$

or simply $2 \alpha \bar{a}$, where $\bar{a}$ is the total amount of capital in the economy. Notice that this is the first-best amount of output and that each unit of capital is used in a project in which the supplied effort is $\alpha$.

Next, note that, without lotteries, at least some units of capital must be used in projects in which the supplied effort is below $\alpha$. From equation (12) above, it is clear that, without lotteries, assigned effort can be $\alpha$ for agents with wealth $a \leq 1$ only if:

$$
\frac{\alpha}{2}\left[1+\sqrt{1-\frac{2 \rho}{\alpha}(1-a)}\right]=\alpha
$$

This is true iff:

$$
\rho(1-a)=0 .
$$

In other words, assigned effort without input lotteries is less than $\alpha$ unless either $\rho=0$ or $a \geq 1$. Because the minimum possible interest rate is $\rho_{\min }=\alpha / 2$, which is greater than zero, this means that it is impossible to assign agents with wealth below unity an effort of $\alpha$. If there are any agents with wealth strictly less than unity, then output must strictly be less than $2 \alpha \bar{a}$.

\section{Proof of Proposition 3}

From proposition 1 above we know that the equilibrium interest rate must be $\alpha$; further, from equation (13) we know that the expected utility of an agent of wealth $a$ is $a \alpha$. 
The planner must also choose contracts which lie in $\mathbf{X}$. Here, the planner's problem is written with the incentive compatibility constraint and the Bayes' compatibility constraint explicitly formulated for convenience:

$$
\max _{\pi^{a} \in L} \sum_{a} \psi^{a} \sum_{\tau q z k} \pi^{a}(\tau, q, z, k)(q-\tau), \text { subject to: }
$$

$$
\begin{aligned}
\sum_{\tau q z k} \pi^{a}(\tau, q, z, k) U_{\tau z} & =\omega(a), \text { all } a \text { in } \mathbf{A}, \\
\sum_{a} \psi^{a}\left[\left(\sum_{\tau q z k} \pi^{a}(\tau, q, z, k) k\right)-a\right] & \geq 0, \\
\sum_{\tau q} \pi^{a}(\tau, q, \bar{z}, \bar{k})\left[U_{\tau \bar{z}}-\frac{P(q \mid \hat{z}, \bar{k})}{P(q \mid \bar{z}, \bar{k})} U_{\tau \hat{z}}\right] & \geq 0,
\end{aligned}
$$

all $\bar{z}, \hat{z}, \bar{k}$ in $\mathbf{Z} \times \mathbf{Z} \times \mathbf{K}$, and the final constraint:

$$
\sum_{\tau} \pi^{a}(\tau, \bar{q}, \bar{z}, \bar{k})=P(\bar{q} \mid \bar{z}, \bar{k}) \sum_{\tau q} \pi^{a}(\tau, q, \bar{z}, \bar{k})
$$

Now replace these choice variables with the familiar choices:

$$
\left\{\xi(a), \tau_{0}(a), \bar{\tau}(a), \underline{\tau}(a)\right\}_{a \in \mathbf{A}},
$$

used in the equilibrium analysis above. As before, we replace the incentive compatibility constraint (P3) with the condition that assigned effort be equal to $\alpha(\bar{\tau}-$ $\tau) / 2$. The policies are Bayes compatible with the underlying probability distribution if the high output is taken to occur with probability $\xi z$.

Imagine that the planner has committed to provide an expected utility of $x$ and no capital with certainty. Let the function $\underline{D}(x)$ be planner's surplus in this case:

$$
\begin{gathered}
\underline{D}(x) \equiv \max _{\tau_{0}}-\tau_{0}, \text { subject to: } \\
\tau_{0}=x .
\end{gathered}
$$

It is easy to see that $\underline{D}(x)=-x$. Now consider the planner's surplus if she assigns 
capital to the agent with certainty:

$$
\begin{gathered}
\bar{D}(x) \equiv \max _{\bar{\tau}, \underline{\tau}, z_{\mathrm{ICC}}} z_{\mathrm{ICC}} \bar{\tau}+\left(1-z_{\mathrm{ICC}}\right) \underline{\tau}, \text { subject to: } \\
z_{\mathrm{ICC}}=\frac{\alpha}{2}(\bar{\tau}-\underline{\tau}) \\
z_{\mathrm{ICC}} \bar{\tau}+\left(1-z_{\mathrm{ICC}}\right) \underline{\tau}-\frac{z_{\mathrm{ICC}}^{2}}{\alpha}=x \\
\underline{\tau}, \bar{\tau} \geq 0
\end{gathered}
$$

For $x \geq 0$ only the non-negativity constraint on $\underline{\tau}$ will bind. Begin by substituting out the incentive compatibility constraint. The constrained optimization problem then becomes:

$$
\begin{aligned}
\bar{D}(x)= & \max _{\bar{\tau}, \underline{\tau}} \mathcal{L}\left(\bar{\tau}, \underline{\tau}, \lambda_{1}, \lambda_{2}\right), \text { where: } \\
\mathcal{L}\left(\bar{\tau}, \underline{\tau}, \lambda_{1}, \lambda_{2}\right)= & \alpha(\bar{\tau}-\underline{\tau})-\alpha(\bar{\tau}-\underline{\tau})^{2}-\underline{\tau}+ \\
& \lambda_{1}\left[\alpha(\bar{\tau}-\underline{\tau})^{2}-\underline{\tau}-x\right]+\lambda_{2} \underline{\tau} .
\end{aligned}
$$

The first-order conditions for maximization require that:

$$
\begin{aligned}
& \lambda_{1}=1-\lambda_{2}, \text { and: } \\
& \lambda_{2}=-1+\sqrt{\alpha / x} .
\end{aligned}
$$

If the non-negativity constraint does not bind, so that $\lambda_{2}=0$, promised utility must satisfy:

$$
x \geq \omega^{\star}, \text { where } \omega^{\star} \equiv \alpha .
$$

Here $\omega^{\star}$ is a "critical utility" which will play an important role later. Thus for agents with promised utility below the critical utility, $x<\omega^{\star}$, we know that the non-negativity constraint on $\underline{\tau}$ will bind. Hence we can split the optimal policies into two sections:

$$
\begin{aligned}
& \underline{\tau}(x)=0, \text { and } \bar{\tau}(x)=2 \sqrt{x / \alpha} \text { if } 0 \leq x \leq \omega^{*}, \\
& \underline{\tau}(x)=x-\omega^{*}, \text { and } \bar{\tau}(x)=x-\omega^{*}+2 \text { if } x \geq \omega^{*} .
\end{aligned}
$$

Thus we can write $\bar{D}(x)$ as:

$$
\begin{aligned}
\bar{D}\left(x \mid 0 \leq x \leq \omega^{*}\right) & =2 \sqrt{\alpha x}-2 x \\
\bar{D}\left(x \mid x \geq \omega^{*}\right) & =\omega^{*}-x .
\end{aligned}
$$




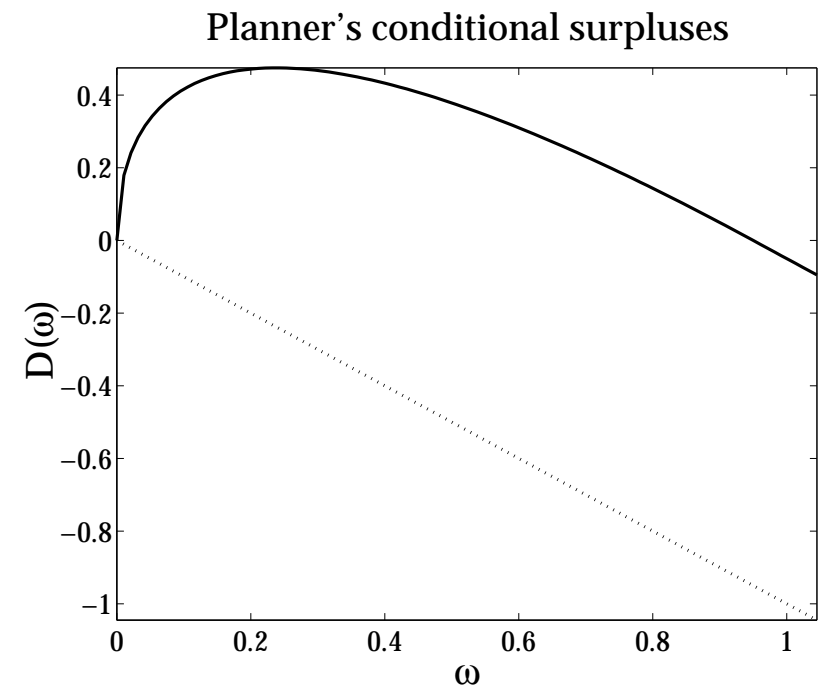

Figure 12: The functions $\bar{D}(\omega)$ (solid) and $\underline{D}(\omega)$ (dotted) when $\alpha=0.5$.

Note that for $x \leq \omega^{*}, \bar{D}$ has an increasing component. This produces the wellknown upward-sloping portion of the Pareto frontier so familiar in models with moral hazard constraints [see e.g. Phelan and Townsend (1991)]. In figure 12 I display typical schedules of $\bar{D}(\omega)$ and $\underline{D}(\omega)$.

We can now completely rewrite the planner's problem:

$$
\begin{gathered}
\xi(a), \underline{\bar{\omega}(a), \underline{\omega}(a)} \sum_{a} \psi^{a}(\xi(a) \bar{D}(\bar{\omega}(a))+(1-\xi(a)) \underline{D}(\underline{\omega}(a))), \\
\text { subject to: } \xi(a) \bar{\omega}(a)+(1-\xi) \underline{\omega}(a)=\omega(a), \\
\sum_{a} \psi^{a} \xi(a)=\bar{a}, \\
\underline{\omega}, \bar{\omega} \geq 0 .
\end{gathered}
$$

Thus the planner can be seen as choosing a joint lottery over capital and utility assignments. Because $\bar{D}(x)>\underline{D}(x)$ the planner finds it cheaper to assign higher utility along with higher capital levels.

The planner's optimal plan will be to set $\underline{\omega}=0, \bar{\omega}=\omega^{*}$ and then adjust $\xi(a)$ to match the promised utility of the agent, so that $\xi(a)=\omega(a) / \omega^{*}$. 
To see this, consider a planner who chooses some values $\underline{\omega}(a), \bar{\omega}(a)$ for an agent with promised utility $\omega(a)$. To satisfy the promise-keeping constraint, $\xi(a)$ must then satisfy:

$$
\xi(a)=f(a) \equiv \frac{\omega(a)-\underline{\omega}(a)}{\bar{\omega}(a)-\underline{\omega}(a)} .
$$

Note that the derivatives of $f$ with respect to $\bar{\omega}$ and $\underline{\omega}$ are:

$$
\frac{\partial f}{\partial \bar{\omega}}=-\frac{f}{\bar{\omega}-\underline{\omega}} \text { and } \frac{\partial f}{\partial \underline{\omega}}=-\frac{1-f}{\bar{\omega}-\underline{\omega}} .
$$

Rewrite the planner's problem (18) in terms of $f$, removing the promise-keeping constraint. Let $\mu$ be the multiplier associated with the resource constraint. The first-order necessary conditions for optimality are:

$$
\begin{aligned}
2 \sqrt{\alpha \bar{\omega}(a)}-\underline{\omega}(a)-(\bar{\omega}(a)-\underline{\omega}(a)) \sqrt{\frac{\alpha}{\bar{\omega}(a)}}=\mu \\
2 \sqrt{\alpha \bar{\omega}(a)}-\bar{\omega}(a)=\mu \\
\sum_{a} \psi^{a} f(a)=\bar{a}
\end{aligned}
$$

Combining equations (19) and (20) immediately produces:

$$
\begin{aligned}
\bar{\omega}(a) & =\alpha, \text { and: } \\
\mu & =\alpha .
\end{aligned}
$$

Notice that the shadow-price of resources, $\mu$, is the equilibrium interest rate, $\mu=$ $\rho^{\star}$. To find $\underline{\omega}(a)$ consider the resource constraint, equation (21):

$$
\sum_{a} \psi^{a} \frac{\omega(a)-\underline{\omega}(a)}{\bar{\omega}(a)-\underline{\omega}(a)}=\sum_{a} \psi^{a} a .
$$

By proposition 1 we know that the competitive equilibrium produces expected utilities:

$$
\begin{aligned}
& \omega(a)=a \alpha, \text { or: } \\
& \omega(a)=a w^{\star} .
\end{aligned}
$$

Combined with the earlier result that $\bar{\omega}(a)=w^{\star}$, the resource constraint becomes:

$$
\sum_{a} \psi^{a} \frac{a w^{\star}-\underline{\omega}(a)}{w^{\star}-\underline{\omega}(a)}=\sum_{a} \psi^{a} a .
$$


Because $a_{\max } \leq 1, \underline{\omega}(a)=0$ all $a$. This in turn provides a policy for $\xi$ :

$$
\xi(a)=a .
$$

So that an agent's wealth determines the probability of getting the capital assignment.

Finally, we must determine the value of the planner's surplus. Because $\bar{\omega}(a)=w^{\star}$ and $\underline{\omega}(a)=0$, and because $\bar{D}\left(w^{\star}\right)=0$ and $\underline{D}(0)=0$, we see immediately that the planner's surplus must be zero. The planner does not have a positive surplus at the expected utilities generated by the competitive equilibrium. Hence the equilibrium allocations must lie on the Pareto frontier.

\section{Proof of Proposition 4}

In this discussion, let $a^{\prime}(a, \rho)$ be the bequest of an agent of wealth $a$ when the interest rate is $\rho$. By assumption, $s \rho<1$, so successful (and rich) agents with wealth $a>1$ bequeath:

$$
a^{\prime}(a \geq 1, \rho \mid \text { success })=s[2+\rho(a-1)]<a .
$$

Unsuccessful (and rich) agents with wealth $a>1$ bequeath:

$$
a^{\prime}(a \geq 1, \rho \mid \text { failure })=s \rho(a-1)<a .
$$

The richest agent in the economy will eventually have wealth $a_{\max }=2 s \leq 1$. From this point forward the interest rate will be $\rho_{\max }$ from proposition 1 above. In figure 13 I display the wealth transitions with lotteries. Agents with wealth below $a_{f}$ will choose to forgo intermediation and use the subsistence technology, where $a_{f}=2 \epsilon / \alpha$.

Now consider the unique invariant distribution to which all initial distributions converge. From the discussion above, it is clear that the richest member of the economy, with wealth $a_{\max }$ will eventually satisfy $a_{\max } \leq 1$ and the equilibrium discussion from above will hold, so that $\rho^{\star}=\rho_{\max }$. Say that this occurs in some period, called, arbitrarily, period $t=0$ and that the distribution of wealth is $\psi_{0}(a)$. Of all agents with wealth $a<a_{f}$, a proportion $\epsilon$ will bequeath $a^{\prime}=2 s$ while the remaining $1-\epsilon$ bequeath $a^{\prime}=0$, and of all agents with wealth $a \geq a_{f}$, a proportion $a \alpha$ will both win the input lottery and have the high output, so bequeathing $a^{\prime}=$ $2 s$ while the remaining $1-a \alpha$ bequeath $a^{\prime}=0$. Thus in the next period, $t=1$, there 


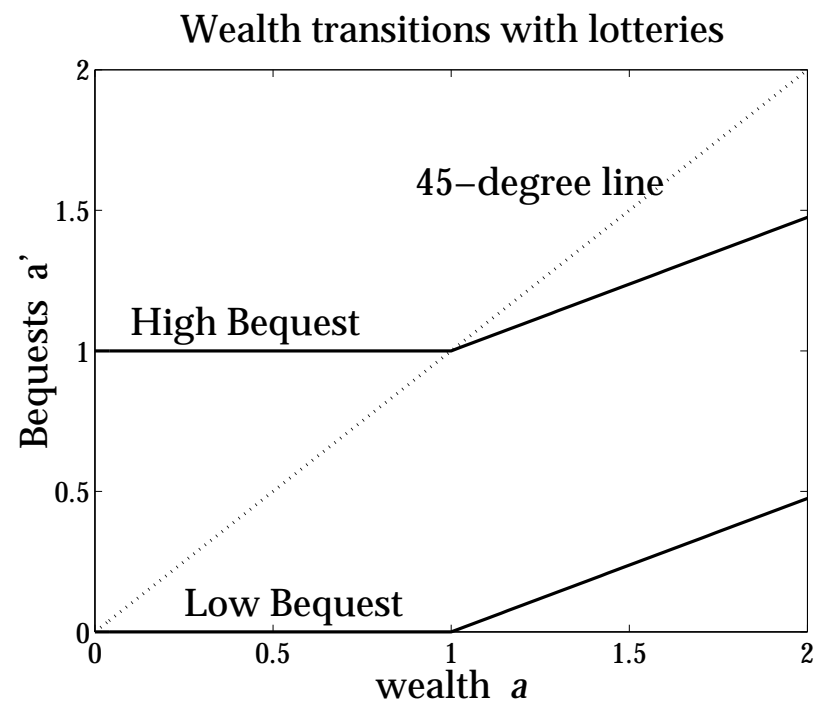

Figure 13: Transitions when $\alpha=0.95, \epsilon=$ 0.05 and $s=0.5$.

will be only two types of agents: agents with wealth 0 and agents with wealth $2 s$. Furthermore, agents will transition only between these two wealth levels. The Markov transition matrix for these states, following Hamilton (1994), is:

$$
\mathcal{P}=\left[\begin{array}{rr}
1-\epsilon & 1-2 \alpha s \\
\epsilon & 2 \alpha s
\end{array}\right] .
$$

Here the columns of $\mathcal{P}$ give today's state while the rows give tomorrow's. Thus, given that an agent has zero wealth, the probability of remaining at zero wealth is $1-\epsilon$ while the probability of transiting to $2 s$ is $\epsilon$. In the same way, given that an agent has $2 s$, the probability of falling to wealth 0 is $1-2 \alpha s$, while the probability of remaining there is $2 \alpha s$.

By solving the eigenproblem associated with $\mathcal{P}$, one can find the eigenvector associated with the unit eigenvalue. This gives the distribution of wealth between the two possible wealth states. It is:

$$
\psi^{\star}=\left(\frac{1-2 \alpha s}{1-2 \alpha s+\epsilon}, \frac{\epsilon}{1-2 \alpha s}\right) .
$$

The aggregate steady-state wealth in this economy is thus $\bar{a}_{\infty}=2 s \epsilon /(1-2 \alpha s+\epsilon)$. 


\section{Numerical Techniques}

Because the objective function (1), the contract constraints (C1), (C2), and (C3) and the zero-profit condition (2) are all linear in the choice objects $y^{a}(\tau, q, z, k)$, for a given set of parameters, wealth $a$ and interest rate $\rho$ it is in principle a simple matter to calculate the optimal contract. Given the optimal contracts and the distribution of wealth at time $t$, one can search for the market-clearing interest rate, $\rho_{t}^{\star}$, and next period's distribution of wealth. I now describe this process in greater detail.

Let $N=n_{T} n_{Q} n_{Z} n_{K}$ be the length of the contract vector. Each position along the vector corresponds to a unique event, that is, a combination of consumption transfer $\tau$, output $q$, effort $z$ and capital $k$. Thus the last entry in the vector might correspond to the event that the highest capital and effort levels are assigned (and used), that the highest output is realized and that the highest possible consumption transfer is made. For concreteness, assume that variables are ordered as $\mathbf{T}, \mathbf{Q}, \mathbf{Z}, \mathbf{K}$, so that the first $n_{T}$ elements of the event space correspond to the events $\left\{\mathbf{T}, q_{1}, z_{1}, k_{1}\right\}$, the next $n_{T}$ elements to $\left\{\mathbf{T}, q_{2}, z_{1}, k_{1}\right\}$ and so on. Let $\mathbf{T}^{e}$ be the $1 \times N$ vector of transfer values at each point in the event space:

$$
\mathbf{T}^{e} \equiv \mathbf{1}_{N-n_{T}}^{\prime} \otimes \mathbf{T}^{\prime}
$$

Here $\mathbf{1}_{m}$ is the $m \times 1$ vector of units and $\otimes$ denotes the Kronecker product. Define $\mathbf{Q}^{e}, \mathbf{Z}^{e}, \mathbf{K}^{e}$ in the same fashion.

\section{Objective and Constraints}

I now describe how to form the linear programming objective vector and constraint matrix for a particular combination of wealth and the interest rate, $(a, \rho)$.

The bank's objective function, equation (1), is the utility of the agent at each point along the event vector. This can be written as:

$$
C_{\mathrm{OBJ}} \equiv u\left(\mathbf{T}^{e}\right)-\theta\left(\mathbf{Z}^{e}\right) .
$$

The bank's zero-profit condition will vary depending on the agent's wealth $a$ and the interest rate under consideration, $\rho$. Thus let:

$$
C_{\mathrm{ZP}}(a, \rho) \equiv \mathbf{Q}^{e}-\mathbf{T}^{e}+\rho\left(a-\mathbf{K}^{e}\right) .
$$


Banks will be required to make zero profits in equilibrium, so let $B_{\mathrm{ZP}}=0$.

Most linear-program solvers easily constrain the choice variables to be positive. To sum over all contract weights, let:

$$
C_{1}=\mathbf{1}_{N}^{\prime}
$$

The contract weights must sum to unity, so let $B_{1}=1$.

The Bayes' consistency constraint, (C2), will take the form of a separate constraint for each $\bar{q}, \bar{z}, \bar{k}$ combination. For a particular combination of inputs and output, let:

$$
C_{2}(\bar{q}, \bar{z}, \bar{k}) \equiv \mathbf{i}(\bar{q}, \bar{z}, \bar{k})-P(\bar{q} \mid \bar{z}, \bar{k}) \mathbf{i}(\bar{z}, \bar{k}) .
$$

Here $\mathbf{i}(\bar{q}, \bar{z}, \bar{k})$ is a $1 \times N$ vector with unit values only where $\mathbf{Q}^{e}=\bar{q}, \mathbf{Z}^{e}=\bar{z}$ and $\mathbf{K}^{e}=\bar{q}$, with zeros elsewhere. $\mathbf{i}(\bar{z}, \bar{k})$ is defined in a similar fashion. There will be $n_{Q} n_{Z} n_{K}$ separate constraints of this form. For each constraint, the right hand side must be zero, so let $B_{2}$ be a column vector of zeros of length $n_{Q} n_{Z} n_{K}$.

Finally, the incentive compatibility constraint, equation (C3), must hold separately for each combination of assigned effort, $\bar{z}$, assigned capital, $\bar{k}$ and potential deviation in effort, $\hat{z}$. For a particular combination $\bar{z}, \bar{k}, \hat{z}$, let:

$$
C_{3}(\bar{z}, \bar{k}, \hat{z})=\mathbf{i}(\bar{z}, \bar{k})\left\{\frac{P(q \mid \hat{z}, \bar{k})}{P(q \mid \bar{z}, \bar{k})}\left[u\left(\mathbf{T}^{e}\right)-\theta(\hat{z})\right]-\left[u\left(\mathbf{T}^{e}\right)-\theta(\bar{z})\right]\right\} .
$$

There will be $n_{Z}^{2} n_{K}$ of these constraints, although several will not be binding (see the discussion above). For a particular input assignment to be incentive compatible, the right hand side must be less than or equal to zero, so let $B_{3}$ be a column-vector of zeros of length $n_{Z}^{2} n_{K}$. These final constraints will be inequality constraints, all others will be equality.

Let $\mathbf{C}(a, \rho)$ be the $M \times N$ matrix of constraints on the linear program when wealth is $a$ and the interest rate is $\rho$. where $M=1+1+n_{Q} n_{Z} n_{K}+n_{Z} n_{K} n_{Z}$. Thus:

$$
\mathbf{C}(a, \rho)=\left(\begin{array}{l}
C_{\mathrm{ZP}} \\
C_{1} \\
C_{2}\left(q_{1}, z_{1}, k_{1}\right) \\
\vdots \\
C_{2}\left(q_{n_{Q}}, z_{n_{Z}}, k_{n_{K}}\right) \\
C_{3}\left(z_{1}, k_{1}, z_{1}\right) \\
\vdots \\
C_{3}\left(z_{n_{Z}}, k_{n_{K}}, z_{n_{Z}}\right)
\end{array}\right) .
$$


In the same way, let $\mathbf{B}$ be the vector of right-hand-size values:

$$
\mathbf{B}=\left(\begin{array}{l}
B_{\mathrm{ZP}} \\
B_{1} \\
B_{2} \\
B_{3}
\end{array}\right) .
$$

\section{Optimal Contracts}

A contract is an $N \times 1$ vector of weights on events. It will be chosen to maximize the objective function, subject to the constraints outlined above. Here, let $\mathbf{y}(a, \rho)$ denote the optimal contract for an agent of wealth $a$ at the interest rate $\rho$. It is formed from the linear program:

$$
\begin{gathered}
\max _{\mathbf{y}(a, \rho)} C_{\text {ОвЈ }} \mathbf{y}(a, \rho), \text { subject to: } \\
\mathbf{C}(a, \rho) \mathbf{y}(a, \rho) \leqq \mathbf{B}
\end{gathered}
$$

Where the first $2+n_{Q} n_{Z} n_{K}$ constraints are equality and the remaining $n_{Z}^{2} n_{K}$ are inequality. Most linear program solvers allow one to specify individual constraints as equality or inequality with ease.

Solving for all wealth levels $a$ in $\mathbf{A}$ at a given interest rate $\rho$ gives the demand for capital by each wealth type. Combined with a distribution of wealth $\psi$ (an $n_{A}$-vector), this implies an aggregate demand for capital:

$$
K^{d}(\rho, \psi)=\sum_{a} \psi^{a}\left[\mathbf{K}^{e} \mathbf{y}(a, \rho)\right]
$$

As above, $\psi^{a}$ is the population mass of wealth $a$.

\section{Equilibrium}

Given a distribution of wealth. $\psi$, equilibrium is a price, $\rho^{\star}$, and a set of contracts for each wealth type, $\mathbf{y}\left(a, \rho^{\star}\right)$, at which aggregate demand and supply of capital are equal and banks are solving their optimization problem, (YP):

$$
\begin{gathered}
K^{d}\left(\rho^{\star}, \boldsymbol{\psi}\right)=\boldsymbol{\psi}^{\prime} \mathbf{A}, \text { and: } \\
\mathbf{y}\left(a, \rho^{\star}\right) \text { solves YP. }
\end{gathered}
$$


It is possible to calculate an upper bound on $\rho^{\star}$, at which no agent, no matter how wealthy, would demand any capital at all. The lower bound may be taken as zero. Thus finding an equilibrium becomes a matter of searching over candidate values of $\rho$ until the capital market clears. At each candidate value, of course, the entire problem (YP) must be solved before the demand for capital may be found. In practice I used combinations of both a grid search (over many predetermined values of $\rho$ ) and a bisection approach.

There is one final fillip to this problem. A bisection algorithm, for example, may converge to a neighborhood of $\rho$ in which, at a candidate value $\rho_{0}$, demand is too high and at a candidate value $\rho_{1}$ demand is too low. The distance between $\rho_{0}$ and $\rho_{1}$ can shrink to the level of machine precision without finding a value of $\rho$ which clears the market. This happens when there is some critical value of $\rho$ at which a large proportion of agents in the economy suddenly switch from demanding capital to not demanding capital. In that case, I set $\rho^{\star}$ to the midpoint of $\rho_{0}$ and $\rho_{1}$ and find a value, $\eta$, such that the convex combination of contracts clears the market. That is, I define:

$$
\mathbf{y}\left(a, \rho^{\star}\right)=\eta \mathbf{y}\left(a, \rho_{0}\right)+(1-\eta) \mathbf{y}\left(a, \rho_{1}\right),
$$

where $\eta$ is such that:

$$
\eta K^{d}\left(\rho_{0}, \boldsymbol{\psi}\right)+(1-\eta) K^{d}\left(\rho_{1}, \boldsymbol{\psi}\right)=\boldsymbol{\psi}^{\prime} \mathbf{A}
$$

This is the standard equilibrium lottery, and is not to be confused with either grid lotteries or input lotteries.

\section{Dynamics}

Given an equilibrium price $\rho^{\star}$ and set of contracts $\mathbf{y}\left(a, \rho^{\star}\right)$, and a starting distribution of wealth, $\psi_{t}$, it is a simple matter to calculate the distribution of wealth in the next period, $\boldsymbol{\psi}_{t+1}$. The assumption that $\mathbf{A}=s \mathbf{T}$ will be particularly useful here. An agent given a consumption transfer of $\tau_{j}$ in $\mathbf{T}$ will bequeath $s \tau_{j}$, which is just (by this assumption) $a_{j}$, the $j$-th element of $\mathbf{A}$. Thus all agents in period $t+1$ born with wealth $a_{j}$ must have had parents who were allocated a consumption transfer of $\tau_{j}$. Finding the mass of agents at $a_{j}$ in period $t+1$ is thus a simple matter of adding up all of the agents allocated consumption transfers of $\tau_{j}$ in period $t$. 
The mass of agents in period $t+1$ with wealth $a_{j}$ in $\mathbf{A}$ will be given by:

$$
\psi_{t+1}^{a_{j}}=\sum_{a} \psi_{t}^{a}\left[\mathbf{i}\left(\tau=\tau_{j}\right) \mathbf{y}_{t}\left(a, \rho_{t}^{\star}\right)\right]
$$

Contracts $\mathbf{y}$ and price $\rho^{\star}$ must now be indexed by time, so that $\mathbf{y}_{t}\left(a, \rho_{t}^{\star}\right)$ is the optimal contract in period $t$ for agents of wealth $a$, at the market clearing interest rate in period $t, \rho_{t}^{\star}$. Repeating this analysis for all possible wealth levels $a$ in $\mathbf{A}$ gives the complete distribution of wealth in period $t, \boldsymbol{\psi}_{t+1}$.

\section{Computations Without Input Lotteries}

The procedure without input lotteries is similar to that outlined above, so I will only briefly cover the differences between the two. When input lotteries cannot be used, optimal contracts between banks and agents are found as the solution to a two-stage algorithm. In the first stage, the bank takes as given the choice of inputs $(z, k)$ and finds the best, feasible, incentive-compatible contracts $\mathbf{y}_{\mathrm{NL}}(a, \rho, z, k)$. If such a contract does not exist (for example, the high effort level, low capital is assigned to an agent with zero wealth) I set the associated expected utility level to a large negative number. I then choose the value of $(z, k)$ associated with the largest expected utility. The optimal contract is thus a pair of objects: an input choice $(z, k)^{\star}$ and an ex post contract over $\mathbf{T}$ and $\mathbf{Q}, \mathbf{y}_{\mathrm{NL}}\left(a, \rho,(z, k)^{\star}\right)$. Everything else then proceeds as described earlier. 


\section{References}

Aghion, P. and P. Bolton (1997). A theory of trickle-down growth and development. Review of Economic Studies 64(2), 151-172.

Bannerjee, A. and A. F. Newman (1993). Occupational choice and the process of development. Journal of Political Economy 59(3), 427-453.

Besley, T., S. Coate, and G. Loury (1993). The economics of rotating savings and credit associations. American Economic Review 83, 792-810.

Besley, T., S. Coate, and G. Loury (1994). Rotating savings and credit associations, credit markets and efficiency. Review of Economic Studies 61(4), 701-19.

Dupor, W. (1998). Complementarities. Ph. D. thesis, University of Chicago.

Ghatak, M. and T. W. Guinnane (1998). The economics of lending with joint liability: A review of theory and practice. Working paper, Yale University.

Hamilton, J. D. (1994). Time Series Analysis. Princeton, New Jersey: Princeton University Press.

Holmström, B. (1982). Moral hazard in teams. Bell Journal of Economics 13(2), 324-340.

Jeong, H. (1998). Decomposition of growth and inequality in Thailand. Manuscript.

Landes, D. S. (1969). The Unbound Prometheus: Technological Change and Industrial Development in Western Europe from 1750 to the Present. Cambridge, UK: Cambridge University Press.

Lehnert, A., E. Ligon, and R. Townsend (1998). Liquidity constraints and incentive contracts. Forthcoming in Macroeconomic Dynamics.

Mansell-Carstens, C. (1993). Popular financial culture in Mexico: The case of the tanda. In L. Randall (Ed.), Changing Structure of Mexico. New York, NY: Sharpe.

Phelan, C. and R. M. Townsend (1991). Computing multi-period, information-constrained optima. Review of Economic Studies 58(5), 853-882. 
Piketty, T. (1997). The dynamics of the wealth distribution and the interest rate with credit rationing. Review of Economic Studies 64(2), 17389.

Prescott, E. and R. Townsend (1984a). General competitive analysis in an economy with private information. International Economic Review 25(1), 1-20.

Prescott, E. and R. Townsend (1984b). Pareto optima and competitive equilibria with adverse selection and moral hazard. Econometrica 52(1), 21-45.

Prescott, E. S. (1998). Computing moral-hazard problems using the Dantzig-Wolfe decomposition algorithm. Federal Reserve Bank of Richmond, Manuscript.

Rosen, S. (1997). Manufacturing inequality. Journal of Labor Economics 15(2), 189-96.

Sadler, M. A. (1998). Escaping poverty: Risk-taking and endogenous inequality in a model of equilibrium growth. Department of Economics, Kansas State University.

Sappington, D. (1983). Limited liability contracts between principal and agent. Journal of Economic Theory 29(2), 1-21. 